\title{
DEFORMATIONS OF SCHEMES AND OTHER BIALGEBRAIC STRUCTURES
}

\author{
J. P. PRIDHAM
}

\begin{abstract}
There has long been a philosophy that every deformation problem in characteristic zero should be governed by a differential graded Lie algebra (DGLA). In this paper, we show how to construct a Simplicial Deformation Complex (SDC) governing any bialgebraic deformation problem. Examples of such problems are deformations of a Hopf algebra, or of an arbitrary scheme. In characteristic zero, SDCs and DGLAs are shown to be equivalent.
\end{abstract}

\section{INTRODUCTION}

In [6], the theory of simplicial deformation complexes (SDCs), reviewed in Section 1, was introduced as an alternative to differential graded Lie algebras (DGLAs), and they were shown to arise naturally from several deformation problems. They can be used to recover the deformation groupoid, are defined in all characteristics, and determine the higher cohomology groups associated to the deformation problem. It was shown how to construct the SDC corresponding to deformations of monadic or comonadic structures. An example of the former is a ring; examples of the latter are co-algebras or sheaves.

The real power of this approach, as developed in Section 2, lies in the ability to construct SDCs from a combination of monadic and comonadic adjunctions. In particular, this gives a systematic approach to defining cohomology theories corresponding to these problems. Examples of this type are deformations of a Hopf algebra, or of a bialgebra. To deform an arbitrary scheme $X$ is equivalent to deforming its structure sheaf $\mathscr{O}_{X}$. The algebra structure of $\mathscr{O}_{X}$ is monadic, while the sheaf structure can be thought of as comonadic.

In Section 3, this idea is applied to provide an SDC for deformations of sheaves of algebras, under more general conditions than those for which a DGLA was constructed by Hinich in [2], namely arbitrary algebras on any site with enough points. By contrast, most previous examples for which DGLAs were constructed were either purely comonadic (e.g. smooth schemes, for which the ring structure does not deform), or purely monadic (e.g. affine schemes, for which the sheaf structure does not deform). For this problem, cohomology of the SDC is just hypercohomology of the dual of Illusie's cotangent complex ([3]). Other examples considered in this section are deformations of subschemes and of group schemes.

Received by the editors October 31, 2005 and, in revised form, April 25, 2006.

2000 Mathematics Subject Classification. Primary 14B12, 14D15, 13D10.

The author was supported during this research by Trinity College, Cambridge and by the Isle of Man Department of Education. 
In Section 4, we see that the localised categories of DGLAs and SDCs are equivalent in characteristic zero. However, while the arrow DGLA $\rightsquigarrow$ SDC is very natural, the arrow SDC $\rightsquigarrow$ DGLA is generally not, which helps to explain why constructing DGLAs is relatively difficult. This does, however, mean that there exist DGLAs governing all the problems described above in characteristic zero.

\section{REVIEW OF SIMPLiCIAL DEFORMATION COMPLEXES AND MONADIC ADJUNCTIONS}

In this section, we summarise the definitions and results of [6, Section 1] (in $\S \S 1.1,1.3$ ), and standard results on monadic adjunctions (in $\S 1.2$ ).

Throughout this paper, we adopt the notation and conventions of [9], so that $\Lambda$ will be a complete local Noetherian ring, with maximal ideal $\mu$ and residue field $k$. $\mathcal{C}_{\Lambda}$ will denote the category of local Artinian $\Lambda$-algebras with residue field $k$. We consider only those functors on $\mathcal{C}_{\Lambda}$ which satisfy $F(k)=\bullet$, the one-point set. We will take [5] as a convenient reference for standard results in deformation theory and adopt its conventions. In particular, a functor $F: \mathcal{C}_{\Lambda} \rightarrow$ Set is called:

(1) homogeneous if $\eta: F\left(B \times_{A} C\right) \rightarrow F(B) \times_{F(A)} F(C)$ is an isomorphism for every $B \rightarrow A$;

(2) a deformation functor if:

(a) $\eta$ is surjective whenever $B \rightarrow A$,

(b) $\eta$ is an isomorphism whenever $A=k$;

(3) smooth if $F(B) \rightarrow F(A)$ whenever $B \rightarrow A$.

Note that a homogeneous functor satisfies Schlessinger's conditions (H1), (H2) and $(\mathrm{H} 4)$, and that a deformation functor satisfies conditions (H1) and (H2).

\subsection{Simplicial deformation complexes.}

Definition 1.1. A simplicial deformation complex $E^{\bullet}$ consists of smooth homogeneous functors $E^{n}: \mathcal{C}_{\Lambda} \rightarrow$ Set for each $n \geq 0$, together with maps

$$
\begin{array}{ll}
\partial^{i}: E^{n} \rightarrow E^{n+1} & 1 \leq i \leq n, \\
\sigma^{i}: E^{n} \rightarrow E^{n-1} & 0 \leq i<n,
\end{array}
$$

an associative product $*: E^{m} \times E^{n} \rightarrow E^{m+n}$, with identity $1: \bullet \rightarrow E^{0}$, where $\bullet$ is the constant functor $\bullet(A)=\bullet$ on $\mathcal{C}_{\Lambda}$, such that:

(1) $\partial^{j} \partial^{i}=\partial^{i} \partial^{j-1} \quad i<j$,

(2) $\sigma^{j} \sigma^{i}=\sigma^{i} \sigma^{j+1} \quad i \leq j$,

(3) $\sigma^{j} \partial^{i}=\left\{\begin{array}{cc}\partial^{i} \sigma^{j-1} & i<j, \\ \text { id } & i=j, i=j+1, \\ \partial^{i-1} \sigma^{j} & i>j+1,\end{array}\right.$

(4) $\partial^{i}(e) * f=\partial^{i}(e * f)$,

(5) $e * \partial^{i}(f)=\partial^{i+m}(e * f)$, for $e \in E^{m}$,

(6) $\sigma^{i}(e) * f=\sigma^{i}(e * f)$,

(7) $e * \sigma^{i}(f)=\sigma^{i+m}(e * f)$, for $e \in E^{m}$.

Definition 1.2. Let $\mathrm{C}^{\bullet}(E)$ be the tangent space of $E^{\bullet}$, i.e. $\mathrm{C}^{n}(E)=E^{n}(k[\epsilon])$.

Definition 1.3. Define the Maurer-Cartan functor $\mathrm{MC}_{E}$ by

$$
\operatorname{MC}_{E}(A)=\left\{\omega \in E^{1}(A): \omega * \omega=\partial^{1}(\omega)\right\} .
$$


In fact, applying $\left(\sigma^{0}\right)^{2}$ to this equation, we see that any such $\omega$ automatically satisfies $\sigma^{0} \omega=1$.

Recall that $E^{0}$ is a group under multiplication. Now, if $\omega \in \mathrm{MC}_{E}(A)$ and $g \in E^{0}(A)$, then $g * \omega * g^{-1} \in \mathrm{MC}_{E}(A)$. We may therefore make the following definition:

\section{Definition 1.4.}

$$
\operatorname{Def}_{E}=\mathrm{MC}_{E} / E^{0}
$$

the quotient being with respect to the adjoint action. The deformation groupoid

$$
\mathfrak{D e f} \mathfrak{f}_{E}
$$

has objects $\mathrm{MC}_{E}$, and morphisms given by $E^{0}$.

Lemma 1.5. For $\omega \in \mathrm{MC}_{E}(A)$, if we let $\partial_{\omega}^{0}(e)=\omega * e$, and $\partial_{\omega}^{n+1}(e)=e * \omega$, for $e \in E^{n}(A)$, then $E^{\bullet}(A)$ becomes a cosimplicial complex.

Definition 1.6. Define the cohomology groups of $E$ to be

$$
\mathrm{H}^{i}(E):=\mathrm{H}^{i}\left(\mathrm{C}^{\bullet}(E)\right),
$$

the cohomology groups of the cosimplicial complex $\mathrm{C}^{\bullet}(E)$.

Theorem 1.7. Def $E_{E}$ is a deformation functor, with tangent space $\mathrm{H}^{1}(E)$ and complete obstruction space $\mathrm{H}^{2}(E)$. For $\omega, \omega^{\prime} \in \operatorname{MC}_{E}(A)$, Iso $\left(\omega, \omega^{\prime}\right)$ is homogeneous, with tangent space $\mathrm{H}^{0}(E)$ and complete obstruction space $\mathrm{H}^{1}(E)$. Moreover, if $\mathrm{H}^{0}(E)=0$, then Def ${ }_{E}$ is homogeneous. If $\phi: E \rightarrow F$ is a morphism of SDCs, and

$$
\mathrm{H}^{i}(\phi): \mathrm{H}^{i}(E) \rightarrow \mathrm{H}^{i}(F)
$$

are the induced maps on cohomology, then:

(1) If $\mathrm{H}^{1}(\phi)$ is bijective, and $\mathrm{H}^{2}(\phi)$ injective, then $\operatorname{Def}_{E} \rightarrow \operatorname{Def}_{F}$ is étale.

(2) If also $\mathrm{H}^{0}(\phi)$ is surjective, then $\operatorname{Def}_{E} \rightarrow \operatorname{Def}_{F}$ is an isomorphism.

(3) Provided condition (1) holds, $\mathfrak{D e f}_{E} \rightarrow \mathfrak{D e f}_{F}$ is an equivalence of functors of groupoids if and only if $\mathrm{H}^{0}(\phi)$ is an isomorphism.

Call a morphism $\phi: E \rightarrow F$ a quasi-isomorphism if the $\mathrm{H}^{i}(\phi): \mathrm{H}^{i}(E) \rightarrow \mathrm{H}^{i}(F)$ are all isomorphisms.

1.2. Review of monadic adjunctions. This section just recalls some standard definitions concerning adjunctions, all of which can be found in [4], and fixes notation which will be used throughout the paper.

Definition 1.8. For categories $\mathcal{D}, \mathcal{E}$, and a pair of functors

$$
\mathcal{D} \underset{F}{\stackrel{G}{\rightleftarrows}} \mathcal{E},
$$

recall that an adjunction $F \dashv G$ is a natural isomorphism

$$
\operatorname{Hom}_{\mathcal{D}}(F A, B) \cong \operatorname{Hom}_{\mathcal{E}}(A, G B) .
$$

We say that $F$ is left adjoint to $G$, or $G$ is right adjoint to $F$. Let $\perp=F G$, and $\top=G F$. To give an adjunction is equivalent to giving two natural transformations, the unit and co-unit,

$$
\eta: \mathrm{id}_{\mathcal{E}} \rightarrow \top, \quad \varepsilon: \perp \rightarrow \mathrm{id}_{\mathcal{D}}
$$

satisfying the triangle identities $\varepsilon F \circ F \eta=\operatorname{id}_{F}, G \varepsilon \circ \eta G=\operatorname{id}_{G}$. 
If a functor has a left adjoint, then it preserves all (inverse) limits. Conversely, provided the categories involved satisfy various additional conditions, the Special Adjoint Functor Theorem ([4], Ch. V.8) proves that any functor which preserves inverse limits has a left adjoint.

Given an adjunction

$$
\mathcal{D}_{\underset{F}{\stackrel{T}{\longrightarrow}} \mathcal{E}}^{\stackrel{U}{\longrightarrow}}
$$

with unit $\eta:$ id $\rightarrow U F$ and co-unit $\varepsilon: F U \rightarrow$ id, we let $\top=U F$, and define the category of $\top$-algebras, $\mathcal{E}^{\top}$, to have objects

$$
\top E \stackrel{\theta}{\rightarrow} E,
$$

such that $\theta \circ \eta_{E}=\mathrm{id}$ and $\theta \circ \top \theta=\theta \circ U \varepsilon_{F E}$.

A morphism

$$
g:\left(\top E_{1} \stackrel{\theta}{\rightarrow} E_{1}\right) \rightarrow\left(\top E_{2} \stackrel{\phi}{\rightarrow} E_{2}\right)
$$

of $\top$-algebras is a morphism $g: E_{1} \rightarrow E_{2}$ in $\mathcal{E}$ such that $\phi \circ \top g=g \circ \theta$.

We define the comparison functor $K: \mathcal{D} \rightarrow \mathcal{E}^{\top}$ by

$$
B \mapsto\left(U F U B \stackrel{U \varepsilon_{B}}{\longrightarrow} U B\right)
$$

on objects, and $K(g)=U(g)$ on morphisms.

Definition 1.9. The adjunction

$$
\mathcal{D} \underset{F}{\stackrel{U}{\underset{F}{\longrightarrow}} \mathcal{E}}
$$

is said to be monadic (also sometimes called tripleable) if $K: \mathcal{D} \rightarrow \mathcal{E}^{\top}$ is an equivalence.

Intuitively, monadic adjunctions correspond to algebraic theories, such as the adjunction

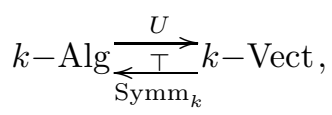

between $k$-algebras and vector spaces over $k, U$ being the forgetful functor. Other examples are Lie algebras or non-commutative algebras, with their respective free functors, over vector spaces, or vector spaces over sets.

Dually, if we have an adjunction

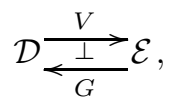

with co-unit $\gamma: V G \rightarrow$ id and unit $\alpha:$ id $\rightarrow G V$, we let $\perp=V G$, and we can define a $\perp$-co-algebra to be a morphism

$$
E \stackrel{\theta}{\rightarrow} \perp E
$$


such that $\gamma_{E} \circ \theta=$ id and $\perp \theta \circ \theta=V \alpha_{G E} \circ \theta$. There is then a natural functor $K: \mathcal{D} \rightarrow \mathcal{E}^{\perp}$ to the category of $\perp$-co-algebras.

Definition 1.10. The adjunction

$$
\mathcal{D} \underset{G}{\stackrel{V}{\stackrel{\perp}{\longrightarrow}} \mathcal{E}}
$$

is said to be comonadic (also sometimes called cotripleable) if $K: \mathcal{D} \rightarrow \mathcal{E}^{\perp}$ is an equivalence.

Finally, the equivalence version of Beck's monadicity theorem gives a criterion for an adjunction to be monadic:

Definition 1.11. A split fork is a diagram

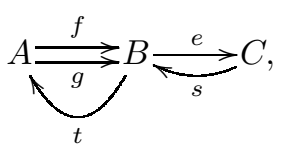

such that $e f=e g$, es $=1, f t=1, g t=s e$. A split coequaliser of $f$ and $g$ is the arrow $e$ of some split fork.

Definition 1.12. A functor $U: \mathcal{D} \rightarrow \mathcal{E}$ is said to reflect coequalisers for a pair $f, g: A \rightarrow B$ if for every diagram $A \underset{g}{\stackrel{f}{\longrightarrow}} B \stackrel{e}{\longrightarrow} C$, with ef $=e g$ and $U e$ a coequaliser of $U f$ and $U g$, the morphism $e$ is a coequaliser of $f$ and $g$.

$U$ is said to reflect isomorphisms if $f$ is an isomorphism whenever $U f$ is. Observe that if $U$ preserves a coequaliser of $f, g$ in $\mathcal{D}$, and $U$ reflects isomorphisms, then $U$ reflects all coequalisers of $f, g$.

Theorem 1.13. The following conditions are equivalent:

(1) The adjunction

$$
\mathcal{D}_{\underset{F}{\stackrel{T}{\rightleftarrows}} \mathcal{E}}^{\stackrel{U}{\longrightarrow}}
$$

is monadic.

(2) If $f, g: D \rightarrow D^{\prime}$ is a parallel pair in $\mathcal{D}$ for which $U f, U g$ has a split coequaliser, then $\mathcal{D}$ has a coequaliser for $f, g$, and $U$ preserves and reflects coequalisers for these pairs.

Proof. This is [4, Ch. VI.7, Ex. 6].

1.3. The SDC associated to a monadic adjunction. Throughout this paper, we will encounter functors $\mathcal{D}: \mathcal{C}_{\Lambda} \rightarrow$ Cat. We will not require that these functors satisfy the condition that $F(k)=\bullet$.

Definition 1.14. Given a functor $\mathcal{D}: \mathcal{C}_{\Lambda} \rightarrow$ Cat, and an object $D \in \operatorname{Ob} \mathcal{D}(k)$, define $\mathfrak{D e f}_{\mathcal{D}, D}: \mathcal{C}_{\Lambda} \rightarrow$ Grpd by setting $\mathfrak{D e f}_{\mathcal{D}, D}(A)$ to be the fibre of $\mathcal{D}(A) \rightarrow \mathcal{D}(k)$ over $(D, \mathrm{id})$.

All the deformation problems we encounter are of this form. 
Definition 1.15. We say a functor $\mathcal{B}: \mathcal{C}_{\Lambda} \rightarrow$ Cat has uniformly trivial deformation theory if

(1) for all $A \in \mathcal{C}_{\Lambda}$ and all $B_{1}, B_{2} \in \mathrm{Ob} \mathcal{B}(A)$, the functor $\operatorname{Mor}_{\mathcal{B}}\left(B_{1}, B_{2}\right): \mathcal{C}_{A} \rightarrow$ Set of morphisms from $B_{1}$ to $B_{2}$ is smooth and homogeneous;

(2) for $A^{\prime} \rightarrow A$ in $\mathcal{C}_{\Lambda}, \mathcal{B}\left(A^{\prime}\right) \rightarrow \mathcal{B}(A)$ is full and essentially surjective.

A typical example of such a functor is that which sends $A$ to the category of flat $A$-modules.

Observe that, if $\mathcal{B}$ is uniformly trivial, then given $B \in \mathrm{Ob} \mathcal{B}(k)$, we may lift it to $\tilde{B} \in \mathrm{Ob} \mathcal{B}(\Lambda)$, and we have an equivalence of functors of groupoids

$$
\left(\tilde{B}, \operatorname{Mor}_{\mathcal{B}}(\tilde{B}, \tilde{B})\right) \stackrel{\sim}{\longrightarrow} \mathfrak{D e f}_{\mathcal{B}, B} .
$$

In this section, we recall the approach used in [6] to construct SDCs, and give some new examples.

Assume we have a monadic adjunction

$$
\mathcal{D} \underset{F}{\stackrel{U}{\underset{F}{\top}}} \mathcal{B},
$$

with unit $\eta:$ id $\rightarrow U F=\top$ and co-unit $\varepsilon: F U \rightarrow$ id, such that $\mathcal{B}$ has uniformly trivial deformation theory. Then, given $D \in \mathcal{D}(k)$, let $B$ be any lift of $U D$ to $\mathcal{B}(\Lambda)$, and define the $\operatorname{SDC} E^{\bullet}$ by

$$
\left.E^{n}=\operatorname{Hom}_{\mathcal{B}}\left(\top^{n} B, B\right)_{U\left(\varepsilon_{D} \circ \ldots \circ \varepsilon_{\perp^{n-1} D}\right.}\right)
$$

the fibre of

$$
\operatorname{Hom}_{\mathcal{B}}\left(\top^{n} B, B\right) \rightarrow \operatorname{Hom}_{\mathcal{B}(k)}\left(\top^{n} B(k), B(k)\right)
$$

over $U\left(\varepsilon_{D} \circ \ldots \circ \varepsilon_{\perp^{n-1} D}\right)$.

We make $E^{\bullet}$ into an SDC by giving it the product $g * h=g \circ \top^{n} h$ for $g \in E^{n}$, and

$$
\begin{aligned}
\partial^{i}(g) & =g \circ \top^{i-1} U \varepsilon_{F \top^{n-i} B}, \\
\sigma^{i}(g) & =g \circ \top^{i} \eta_{\top^{n-i-1} B} .
\end{aligned}
$$

Theorem 1.16. With the notation as above, we have an equivalence of functors of groupoids

$$
\mathfrak{D} \mathfrak{e} \mathfrak{f}_{\mathcal{D}, D} \stackrel{\sim}{\longrightarrow} \mathfrak{D} \mathfrak{e} \mathfrak{f}_{E}
$$

1.3.1. Deformations of sheaves - Godement resolution. Take a sheaf of $k$-vector spaces $\mathscr{M}_{0}$ on a site $X$ with enough points. The deformation functor will associate to $A$ sheaves $\mathscr{M}_{A}$ of flat $A$-modules such that $\mathscr{M}_{A} \otimes k=\mathscr{M}_{0}$, modulo infinitesimal isomorphisms.

Let $X^{\prime}$ be the set of points of $X$. Since $X$ has enough points, the inverse image functor $\operatorname{Shf}(X) \rightarrow \prod_{x \in X^{\prime}} \operatorname{Shf}(x)$ reflects isomorphisms. Explicitly, this says that a morphism $\theta: \mathscr{F} \rightarrow \mathscr{G}$ of sheaves on $X$ is an isomorphism whenever the morphisms $\theta_{x}: \mathscr{F}_{x} \rightarrow \mathscr{G}_{x}$ are for all $x \in X^{\prime}$. In the reasoning which follows, it will suffice to replace $X^{\prime}$ by any subset with this property. 
If we are working on the Zariski site, we may take $X^{\prime}=\coprod_{x \in X} x$. On the étale site, we may take

$$
X^{\prime}=\coprod_{x \in X} \bar{x}
$$

where for each $x \in X$, a geometric point $\bar{x} \rightarrow X$ has been chosen. For Jacobson schemes, we may consider only closed points $x \in X$. We define the category $\operatorname{Shf}\left(X^{\prime}\right)$ by $\operatorname{Shf}\left(X^{\prime}\right):=\prod_{x \in X^{\prime}} \operatorname{Shf}(x)$.

There is an adjunction

$$
\operatorname{Shf}\left(X^{\prime}\right) \underset{u^{*}}{\stackrel{u_{*}}{\underset{\top}{\longrightarrow}}} \operatorname{Shf}(X),
$$

where the maps $u_{x}: x \rightarrow X$ combine to form maps $u^{*}: \operatorname{Shf}(X) \rightarrow \operatorname{Shf}\left(X^{\prime}\right)$, and $u_{*}: \operatorname{Shf}\left(X^{\prime}\right) \rightarrow \operatorname{Shf}(X)$ is given by $u_{*} \mathscr{F}=\prod_{x \in X^{\prime}} u_{x *} \mathscr{F}_{x}$. Let $\alpha_{\mathscr{F}}: \mathscr{F} \rightarrow u_{*} u^{*} \mathscr{F}$ be the unit of the adjunction. Observe that the category of flat $A$-modules on $X^{\prime}$ has uniformly trivial deformation theory.

It follows from Theorem 1.13 that this adjunction is comonadic, and from Theorem 2.2, the SDC governing this problem is

$$
E^{n}=\operatorname{Hom}_{\Lambda}\left(\mathscr{N},\left(u^{*} u_{*}\right)^{n}(\mathscr{N})\right)_{\alpha^{n}},
$$

where $\mathscr{N}$ is a flat $\mu$-adic $\Lambda$-module on $X^{\prime}$ with $\mathscr{N} \otimes k=u^{*} \mathscr{M}_{0}$, and $\alpha^{n}=$ $u^{*}\left(\alpha_{\left(u_{*} u^{*}\right)^{n-1} \mathscr{M}_{0}} \circ \ldots \circ \alpha_{\mathscr{M}_{0}}\right)$.

Defining a sheaf of SDCs on $X$ by

$$
\mathscr{E}^{n}=u_{*} \operatorname{hom}_{\Lambda}\left(\mathscr{N},\left(u^{*} u_{*}\right)^{n}(\mathscr{N})\right)_{\alpha^{n}},
$$

we get, as in $[6, \S 1.2 .2]$,

$$
\mathrm{H}^{i}(E)=\mathrm{H}^{i}\left(\mathrm{C}^{\bullet}(E)\right)=\mathbb{H}^{i}\left(X, \mathrm{C}^{\bullet}(\mathscr{E})\right)=\operatorname{Ext}_{k}^{i}\left(\mathscr{M}_{0}, \mathscr{M}_{0}\right),
$$

as expected.

1.3.2. Deformations of co-algebras. Given a flat (co-associative) co-algebra (with co-unit) $C_{0} / k$, we wish to create an SDC describing flat deformations $C_{A} / A$ such that $C_{A} \otimes_{A} k=C_{0}$, modulo infinitesimal isomorphisms.

There is, up to isomorphism, a unique flat $\mu$-adic $\Lambda$-module $M$ such that $M \otimes_{\Lambda} k$ $=C_{0}$. There is an adjunction

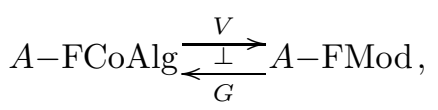

between the category of flat co-algebras over $A$, and the category of flat modules over $A$, where $V$ is the forgetful functor and the free functor $G$ exists by the Special Adjoint Functor Theorem (since $A$-FCoAlg has all colimits, and $V$ preserves these). Note that in this case the free functor is hard to write down explicitly, but this is unnecessary for our purposes. See [10] for such a description.

By Theorem 1.13, this adjunction is comonadic, so that deformations of $C_{0}$ are given by the SDC

$$
\left.E^{n}=\operatorname{Hom}_{\Lambda}\left(M, \perp^{n} M\right)_{U\left(\alpha_{\top n-1} C_{0}\right.}{ }^{\ldots} \ldots \circ \alpha_{C_{0}}\right) .
$$




\section{Bialgebraic Deformations}

In this section we make formal the approach which has been used so far to compute SDCs. The idea is that we throw away properties of the object which we wish to deform, until we obtain something whose deformations are trivial.

In general, we will not be able to pass from the category $\mathcal{D}$ to a category $\mathcal{B}$ with uniformly trivial deformation theory via a single monadic or comonadic adjunction. However, we should be able to pass from $\mathcal{D}$ to some $\mathcal{B}$ via a chain of monadic and comonadic adjunctions.

Not only should we have monadic and comonadic adjunctions, but, informally, the forgetful functors should commute with one another. More precisely, assume that we have a diagram

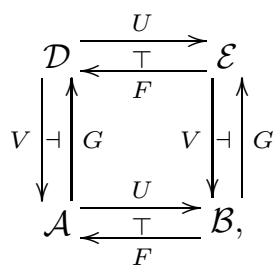

where $\mathcal{B}$ has uniformly trivial deformation theory, with $F \dashv U$ monadic and $G \vdash V$ comonadic. Let

$$
\begin{array}{ll}
\top_{\mathrm{h}}=U F, & \perp_{\mathrm{h}}=F U, \\
\perp_{\mathrm{v}}=V G, & \top_{\mathrm{v}}=G V,
\end{array}
$$

with

$$
\eta: 1 \rightarrow \top_{\mathrm{h}}, \quad \gamma: \perp_{\mathrm{v}} \rightarrow 1, \quad \varepsilon: \perp_{\mathrm{h}} \rightarrow 1 \text { and } \alpha: 1 \rightarrow \top_{\mathrm{v}} .
$$

The commutativity condition is that the following identities hold (up to canonical isomorphism):

$$
\begin{array}{r}
G U=U G \quad \text { or } \quad F V=V F, \\
U V=V U, \\
V \varepsilon=\varepsilon V \quad \text { or } \quad U \alpha=\alpha U, \\
V \eta=\eta V \quad \text { or } \quad U \gamma=\gamma U .
\end{array}
$$

Observe that the adjoint properties ensure that identities on the same line are equivalent.

Lemma 2.1. For $E \in \mathcal{E}, A \in \mathcal{A}$, consider the diagram of isomorphisms given by the adjunctions:

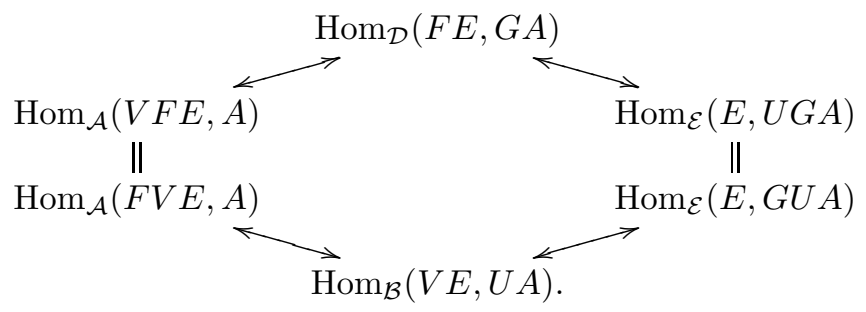

The identities (1)-(4) ensure that all squares in this diagram commute. In particular, for any $B \in \mathcal{B}$, the map

$$
\eta_{B} \circ \gamma_{B}: V G B \rightarrow U F B
$$


corresponds to a map

$$
\rho_{B}=\varepsilon_{G F B} \circ F G \eta_{B}=G F \gamma_{B} \circ \alpha_{F G B}: F G B \rightarrow G F B,
$$

with

$$
\begin{aligned}
\gamma_{T_{\mathrm{h}} B} \circ U V\left(\rho_{B}\right) \circ \eta_{\perp_{\mathrm{v}} B} & =\eta_{B} \circ \gamma_{B}, \\
U\left(\rho_{B}\right) \circ \eta_{G B} & =G\left(\eta_{B}\right), \\
\gamma_{F B} \circ V\left(\rho_{B}\right) & =F\left(\gamma_{B}\right) .
\end{aligned}
$$

By the naturality of $\varepsilon: \perp_{\mathrm{h}} \rightarrow \mathrm{id}$, we have $\varepsilon \circ\left(\perp_{\mathrm{h}} \varepsilon\right)=\varepsilon \circ\left(\varepsilon \perp_{\mathrm{h}}\right)$, so we obtain a canonical map $\varepsilon^{n}: \perp_{\mathrm{h}}^{n} \rightarrow \mathrm{id}$, given by any such composition of $\varepsilon$ 's. [For instance

$$
\varepsilon^{n}=\varepsilon \circ\left(\perp_{\mathrm{h}} \varepsilon\right) \circ \ldots \circ\left(\perp_{\mathrm{h}}^{n-1} \varepsilon\right)
$$

is one such composition.] We have similar maps for each of the units and co-units, giving:

$$
\begin{array}{rlrl}
\varepsilon^{n}: \perp_{\mathrm{h}}^{n} & \rightarrow \mathrm{id}, & \eta^{n}: \mathrm{id} & \rightarrow \top_{\mathrm{h}}^{n}, \\
\alpha^{n}: \mathrm{id} & \rightarrow \mathrm{T}_{\mathrm{v}}^{n}, & \gamma^{n}: \perp_{\mathrm{v}}^{n} \rightarrow \mathrm{id} .
\end{array}
$$

Let

$$
\delta=U V(\rho): \top_{\mathrm{h}} \perp_{\mathrm{v}} \rightarrow \perp_{\mathrm{v}} \top_{\mathrm{h}} .
$$

Since $\delta$ is natural,

$$
\left(\delta \perp_{\mathrm{v}} \top_{\mathrm{h}}\right) \circ\left(\top_{\mathrm{h}} \perp_{\mathrm{v}} \delta\right)=\left(\perp_{\mathrm{v}} \top_{\mathrm{h}} \delta\right) \circ\left(\delta \top_{\mathrm{h}} \perp_{\mathrm{v}}\right) .
$$

Therefore any composition of $\delta$ 's gives us the same canonical map

$$
\delta^{m, n}: \top_{\mathrm{h}}^{m} \perp_{\mathrm{v}}^{n} \rightarrow \perp_{\mathrm{v}}^{n} \top_{\mathrm{h}}^{m} .
$$

Theorem 2.2. Suppose we have a diagram as above. Then, for $D \in \operatorname{Ob} \mathcal{D}(k)$, let $B$ be any lift of $U V D \in \mathrm{Ob} \mathcal{B}(k)$ to $\mathrm{Ob} \mathcal{B}(\Lambda)$ (by the hypothesis on $\mathcal{B}$, such a lift must exist and be unique up to isomorphism). Set

$$
E^{n}=\operatorname{Hom}_{\mathcal{B}}\left(\top_{\mathrm{h}}^{n} B, \perp_{\mathrm{v}}^{n} B\right)_{U V\left(\alpha_{D}^{n} \circ \varepsilon_{D}^{n}\right)} .
$$

We give $E^{\bullet}$ the multiplication

$$
g * h=\perp_{\mathrm{v}}^{n}(g) \circ \delta^{m, n} \circ \top_{\mathrm{h}}^{m}(h),
$$

for $g \in E^{m}, h \in E^{n}$, and

$$
\begin{aligned}
& \partial^{i}(g)=\perp_{\mathrm{v}}^{i-1} V \alpha_{G \perp_{\mathrm{v}}^{m-i} B} \circ g \circ \top_{\mathrm{h}}^{i-1} U \varepsilon_{F \top_{\mathrm{h}}^{m-i} B}, \\
& \sigma^{i}(g)=\perp_{\mathrm{v}}^{i} \gamma_{\perp_{\mathrm{v}}^{m-i-1} B} \circ g \circ \top_{\mathrm{h}}^{i} \eta_{\top_{\mathrm{h}}^{m-i-1} B} .
\end{aligned}
$$

Then

$$
\mathfrak{D e f} \mathfrak{f}_{\mathcal{D}, D} \simeq \mathfrak{D e} \mathfrak{f}_{E}
$$

Proof. First observe that this is, indeed, an SDC. We need to check that the product is associative. For $f \in S^{l}, g \in S^{m}, h \in S^{n}$, we have

$$
\begin{aligned}
f *(g * h) & =\perp_{\mathrm{v}}^{m+n} f \circ \delta^{l, m+n} \circ \top_{\mathrm{h}}^{l}\left(\perp_{\mathrm{v}}^{n} g \circ \delta^{m, n} \circ \top_{\mathrm{h}}^{m} h\right) \\
& =\perp_{\mathrm{v}}^{m+n} f \circ\left(\perp_{\mathrm{v}}^{n} \delta^{l, m} \circ \delta^{l, n} \perp_{\mathrm{v}}^{m}\right) \circ \top_{\mathrm{h}}^{l} \perp_{\mathrm{v}}^{n} g \circ \top_{\mathrm{h}}^{l} \delta^{m, n} \circ \top_{\mathrm{h}}^{l+m} h \\
& =\perp_{\mathrm{v}}^{m+n} f \circ \perp_{\mathrm{v}}^{n} \delta^{l, m} \circ \perp_{\mathrm{v}}^{n} \top_{\mathrm{h}}^{l} g \circ \delta^{l, n} \top_{\mathrm{h}}^{m} \circ \top_{\mathrm{h}}^{l} \delta^{m, n} \circ \top_{\mathrm{h}}^{l+m} h \\
& =\perp_{\mathrm{v}}^{n}\left(\perp_{\mathrm{v}}^{m} f \circ \delta^{l, m} \circ \top_{\mathrm{h}}^{l} g\right) \circ \delta^{l+m, n} \circ \top_{\mathrm{h}}^{l+m} h \\
& =(f * g) * h .
\end{aligned}
$$


We now have a lemma:

Lemma 2.3. To give a map $f: \top_{\mathrm{h}} B \rightarrow \perp_{\mathrm{v}} B$ satisfying the Maurer-Cartan equation

$$
\perp_{\mathrm{v}} f \circ \delta \circ \top_{\mathrm{h}} f=V \alpha_{G B} \circ f \circ U \varepsilon_{F B}
$$

is the same as giving maps $\theta: \top_{\mathrm{h}} B \rightarrow B$ and $\phi: B \rightarrow \perp_{\mathrm{v}} B$ satisfying the MaurerCartan equations:

$$
\begin{aligned}
\theta \circ \top_{\mathrm{h}} \theta & =\theta \circ U \varepsilon_{F B}, \\
\perp_{\mathrm{v}} \phi \circ \phi & =V \alpha_{G B} \circ \phi,
\end{aligned}
$$

and the compatibility condition

$$
\perp_{\mathrm{v}} \theta \circ \delta_{B} \circ \top_{\mathrm{h}} \phi=\phi \circ \theta .
$$

Proof of lemma. Given such an $f$, we have

$$
\begin{aligned}
f & =\gamma_{\perp_{\mathrm{v}} B} \circ V \alpha_{G B} \circ f \circ U \varepsilon_{F B} \circ \eta_{\top_{\mathrm{h}} B} \\
& =\gamma_{\perp_{\mathrm{v}} B} \circ \perp_{\mathrm{v}} f \circ \delta_{B} \circ \top_{\mathrm{h}} f \circ \eta_{\top_{\mathrm{h}} B} \\
& =f \circ \gamma_{T_{\mathrm{h}} B} \circ \delta_{B} \circ \eta_{\perp_{\mathrm{v}} B} \circ f \\
& =f \circ \eta_{B} \circ \gamma_{B} \circ f .
\end{aligned}
$$

Let $\phi=f \circ \eta_{B}$, and $\theta=\gamma_{B} \circ f$. Now,

$$
\begin{aligned}
\gamma_{B} \circ \gamma_{\perp_{\mathrm{v}} B} \circ \perp_{\mathrm{v}} f \circ \delta_{B} \circ T_{\mathrm{h}} f & =\gamma_{B} \circ \gamma_{\perp_{\mathrm{v}} B} \circ V \alpha_{G B} \circ f \circ U \varepsilon_{F B}, \\
\gamma_{B} \circ f \circ \gamma_{T_{\mathrm{h}} B} \circ \delta_{B} \circ \top_{\mathrm{h}} f & =\gamma_{B} \circ f \circ U \varepsilon_{F B}, \\
\gamma_{B} \circ f \circ U\left(\gamma_{F B} \circ V\left(\rho_{B}\right)\right) \circ \top_{\mathrm{h}} f & =\gamma_{B} \circ f \circ U \varepsilon_{F B}, \\
\gamma_{B} \circ f \circ \top_{\mathrm{h}} \gamma_{B} \circ T_{\mathrm{h}} f & =\gamma_{B} \circ f \circ U \varepsilon_{F B}, \\
\theta \circ \top_{\mathrm{h}} \theta & =\theta \circ U \varepsilon_{F B},
\end{aligned}
$$

using the Maurer-Cartan equations and Lemma 2.1. Similarly we obtain the Maurer-Cartan equation for $\phi$, and finally

$$
\begin{aligned}
\perp_{\mathrm{v}} \theta \circ \delta_{B} \circ \top_{\mathrm{h}} \phi & =\perp_{\mathrm{v}} \gamma_{B} \circ \perp_{\mathrm{v}} f \circ \delta_{B} \circ \top_{\mathrm{h}} f \circ \top_{\mathrm{h}} \eta_{B} \\
& =\perp_{\mathrm{v}} \gamma_{B} \circ V \alpha_{G B} \circ f \circ U \varepsilon_{F B} \circ \top_{\mathrm{h}} \eta_{B} \\
& =f \\
& =\phi \circ \theta .
\end{aligned}
$$

Conversely, given such $\theta$ and $\phi$, set $f=\phi \circ \theta$. We obtain:

$$
\begin{aligned}
\perp_{\mathrm{v}} f \circ \delta_{B} \circ \top_{\mathrm{h}} f & =\perp_{\mathrm{v}} \phi \circ \perp_{\mathrm{v}} \theta \circ \delta_{B} \circ \top_{\mathrm{h}} \phi \circ \top_{\mathrm{h}} \theta \\
& =\perp_{\mathrm{v}} \phi \circ \phi \circ \theta \circ \top_{\mathrm{h}} \theta \\
& =V \alpha_{G B} \circ \phi \circ \theta \circ U \varepsilon_{F B} \\
& =V \alpha_{G B} \circ f \circ U \varepsilon_{F B} .
\end{aligned}
$$

Recall that every element $f$ of $\mathrm{MC}_{E}(A)$ must satisfy $\sigma^{0}(f)=1$, which in this case is $\gamma_{B} \circ f \circ \eta_{B}=\mathrm{id}$. This means that the pair $(\theta, \phi)$ above satisfies $\theta \circ \eta_{B}=\mathrm{id}$ and $\gamma_{B} \circ \phi=$ id. 
With the notation of $\S 1.2$, we obtain an adjunction

$$
\begin{aligned}
& \mathcal{E}^{\top_{\mathrm{h}}} \stackrel{V}{\stackrel{\perp}{\rightleftarrows}} \mathcal{B}^{\top_{\mathrm{h}}}, \\
&\left(\top_{\mathrm{h}} E \stackrel{f}{\longrightarrow} E\right) \mapsto\left(\top_{\mathrm{h}} V E \stackrel{V f}{\longrightarrow} V E\right), \\
&\left(\top_{\mathrm{h}} G B \stackrel{G(g) \circ U\left(\rho_{B}\right)}{\longrightarrow} G B\right) \longleftrightarrow\left(\top_{\mathrm{h}} B \stackrel{g}{\rightarrow} B\right),
\end{aligned}
$$

and such a pair $(\theta, \phi)$ is precisely the same as an element of $\left(\mathcal{B}^{\top_{h}}\right)^{\perp_{\mathrm{v}}}$. We also have a correspondence between the action of $E^{0}$ on $\mathrm{MC}_{E}$ and morphisms in $\left(\mathcal{B}^{\top_{\mathrm{h}}}\right)^{\perp_{\mathrm{v}}}$, so that $\mathfrak{D} \mathfrak{e f} E$ corresponds to the fibre of

$$
\left(\mathcal{B}^{\top_{\mathrm{h}}}\right)^{\perp_{\mathrm{v}}} \rightarrow\left(\mathcal{B}^{\top_{\mathrm{h}}}\right)^{\perp_{\mathrm{v}}}(k)
$$

over the image of $(D, \mathrm{id})$.

Now, the equivalence

$$
\begin{aligned}
K: \mathcal{A} & \rightarrow \mathcal{B}^{T_{\mathrm{h}}} \\
A & \mapsto\left(\top_{\mathrm{h}} U A \stackrel{U \varepsilon_{A}}{\longrightarrow} U A\right)
\end{aligned}
$$

arising from the monadic adjunction $F \dashv U$ satisfies

$$
\begin{aligned}
\perp_{\mathrm{v}} K A & =V G\left(\top_{\mathrm{h}} U A \stackrel{U \varepsilon_{A}}{\longrightarrow} U A\right) \\
& =\left(\top_{\mathrm{h}} \perp_{\mathrm{v}} U A \stackrel{\perp_{\mathrm{v}} U \varepsilon_{A} \circ V U\left(\rho_{U A}\right)}{\longrightarrow} \perp_{\mathrm{v}} U A\right) .
\end{aligned}
$$

Moreover,

$$
\begin{aligned}
\perp_{\mathrm{v}} U \varepsilon_{A} \circ V U\left(\rho_{U A}\right) & =U V\left(G \varepsilon_{A} \circ\left(\varepsilon_{G F U A} \circ F G \eta_{U A}\right)\right) \\
& =U V\left(\left(\varepsilon_{G A} \circ F U G \varepsilon_{A}\right) \circ F G \eta_{U A}\right) \\
& =U V\left(\varepsilon_{G A} \circ F G\left(U \varepsilon_{A} \circ \eta_{U A}\right)\right) \\
& =U V\left(\varepsilon_{G A}\right),
\end{aligned}
$$

so

$$
\perp_{\mathrm{v}} K A=\left(\top_{\mathrm{h}} \perp_{\mathrm{v}} U A \stackrel{U V\left(\varepsilon_{G A}\right)}{\longrightarrow} \perp_{\mathrm{v}} U A\right)=K \perp_{\mathrm{v}} A .
$$

Since $K$ is an equivalence, this induces an equivalence of categories

$$
\begin{aligned}
\mathcal{A}^{\perp_{\mathrm{v}}} & \stackrel{K_{\mathrm{h}}}{\longrightarrow}\left(\mathcal{B}^{\top_{\mathrm{h}}}\right)^{\perp_{\mathrm{v}}} \\
\left(A \stackrel{f}{\longrightarrow} \perp_{\mathrm{v}} A\right) & \mapsto\left(K A \stackrel{K f}{\longrightarrow} \perp_{\mathrm{v}} K A\right) .
\end{aligned}
$$

But $G \vdash V$ is comonadic, so

$$
\mathcal{D} \stackrel{K_{\mathrm{v}}}{\longrightarrow} \mathcal{A}^{\perp_{\mathrm{v}}}
$$

is an equivalence. Thus we have an equivalence of groupoids

$$
\mathfrak{D e f}_{\mathcal{D}, D} \stackrel{\sim}{\longrightarrow} \mathfrak{D e f}_{\mathcal{A}^{\perp \mathrm{v}}, K_{\mathrm{v}} D} \stackrel{\sim}{\longrightarrow} \mathfrak{D e f}_{\left(\mathcal{B}^{\top} \mathrm{h}\right)}{ }^{\perp_{\mathrm{v}}}, K_{\mathrm{h}} K_{\mathrm{v}} D=\mathfrak{D e f}_{E} .
$$

Given a category $\mathcal{D}$, we now need a procedure for finding a category $\mathcal{B}$ with uniformly trivial deformation theory. We do this by thinking of the functors $U$ and $V$ as being forgetful functors, and looking for structures in $\mathcal{D}$ to discard.

Definition 2.4. Given a category $\mathcal{B}: \mathcal{C}_{\Lambda} \rightarrow$ Cat, define a set of structures $\Sigma$ over $\mathcal{B}$ to consist of the following data:

(1) a finite set $\Sigma=\Sigma^{+} \sqcup \Sigma^{-}$, 
(2) for each subset $S \subset \Sigma$, a category $\mathcal{B}^{S}$, with $\mathcal{B}^{\emptyset}=\mathcal{B}$,

(3) (a) for each $s \in \Sigma^{+}$and each $S \subset \Sigma$ not containing $s$, a monadic adjunction

$$
\mathcal{B}^{S \cup\{s\}} \underset{F_{s}}{\stackrel{U_{s}}{\underset{T}{\longrightarrow}}} \mathcal{B}^{S},
$$

(b) for each $s \in \Sigma^{-}$and each $S \subset \Sigma$ not containing $s$, a comonadic adjunction

$$
\mathcal{B} S \cup\{s\} \underset{G_{s}}{\stackrel{V_{s}}{\rightleftarrows}} \mathcal{B}^{S},
$$

satisfying the commutativity conditions given below.

For $s \in \Sigma^{+}$we write

$$
\top_{s}=U_{s} F_{s} \quad \text { and } \quad \perp_{s}=F_{s} U_{s}
$$

with

$$
\eta_{s}: 1 \rightarrow \top_{s} \quad \text { and } \quad \varepsilon_{s}: \perp_{s} \rightarrow 1
$$

For $s \in \Sigma^{-}$we write

$$
\perp_{s}=V_{s} G_{s} \quad \text { and } \quad \top_{s}=G_{s} V_{s}
$$

with

$$
\gamma_{s}: \perp_{s} \rightarrow 1 \text { and } \alpha_{s}: 1 \rightarrow \top_{s} .
$$

The commutativity conditions are that the following should hold:

(1) For each distinct pair $s_{1}, s_{2} \in \Sigma^{+}$, and each $S \subset \Sigma$ not containing $s_{1}, s_{2}$, the morphisms in the diagram

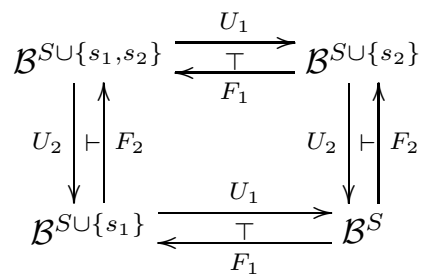

satisfy

$$
\begin{gathered}
F_{1} F_{2}=F_{2} F_{1} \quad \text { or } \quad U_{1} U_{2}=U_{2} U_{1}, \\
U_{1} \varepsilon_{2}=\varepsilon_{2} U_{1}, \quad U_{2} \varepsilon_{1}=\varepsilon_{1} U_{2}, \\
U_{1} \eta_{2}=\eta_{2} U_{1}, \quad U_{2} \eta_{1}=\eta_{1} U_{2} .
\end{gathered}
$$

(2) For each distinct pair $s_{1}, s_{2} \in \Sigma^{-}$, and each $S \subset \Sigma$ not containing $s_{1}, s_{2}$, the morphisms in the diagram

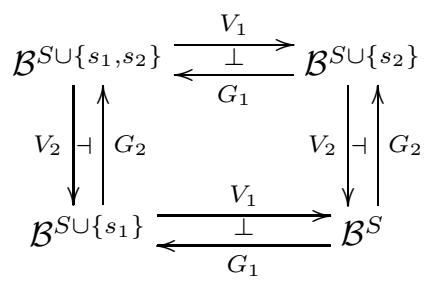


satisfy

$$
\begin{gathered}
G_{1} G_{2}=G_{2} G_{1} \quad \text { or } \quad V_{1} V_{2}=V_{2} V_{1}, \\
V_{1} \alpha_{2}=\alpha_{2} V_{1}, \quad V_{2} \alpha_{1}=\alpha_{1} V_{2}, \\
V_{1} \gamma_{2}=\gamma_{2} V_{1}, \quad V_{2} \gamma_{1}=\gamma_{1} V_{2} .
\end{gathered}
$$

(3) For each $s_{1} \in \Sigma^{+}$and $s_{2} \in \Sigma^{-}$, and each $S \subset \Sigma$ not containing $s_{1}, s_{2}$, the morphisms in the diagram

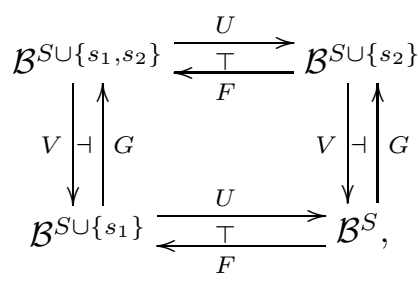

satisfy

$$
\begin{array}{r}
G U=U G \quad \text { or } \quad F V=V F, \\
U V=V U, \\
V \varepsilon=\varepsilon V \quad \text { or } \quad U \alpha=\alpha U, \\
V \eta=\eta V \quad \text { or } \quad U \gamma=\gamma U .
\end{array}
$$

Lemma 2.5. Given a set of structures $\Sigma$ over a category $\mathcal{B}$, we have a diagram

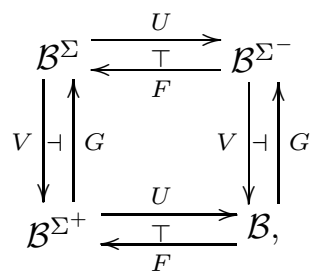

satisfying the equations (1)-(4) on p. 1608, with $F \dashv U$ monadic and $G \vdash V$ comonadic.

Proof. We define $F$ (resp. $U$ ) to be the composition of the $F_{s}$ (resp $U_{s}$ ) for all $s \in \Sigma^{+}$, noting that the order of composition does not matter, since these functors commute with one another. We define $G$ and $V$ analogously. It is immediate that $F \dashv U$ and $G \vdash V$ are adjoint pairs, and that the commutativity conditions (1)-(4) are satisfied.

It thus remains only to show that $F \dashv U$ is monadic, and $G \vdash V$ comonadic. This can be done by using a similar approach to that used in the proof of Theorem 2.2. The statement is that $\mathcal{B}^{\Sigma^{+}} \simeq \mathcal{B}^{\top+}$, where $\top_{+}=F U$, which is proved by induction on the cardinality of $\Sigma^{+}$.

Remarks 2.6. (1) This lemma shows that every set of structures can be replaced by a set of at most two elements, so it might seem that introducing the notion of a set of structures was not helpful. However, it is frequently easier to find the individual adjunctions than the composites, as will be seen in later examples (notably the deformation of a group scheme in Section $3.4)$. 
(2) For most of the deformation problems for which DGLAs have previously been constructed, it seems that there is a set of structures which is either wholly monadic or wholly comonadic.

\section{EXAMPLES}

3.1. Deformations of Hopf algebras. Given a flat (associative, commutative, co-associative) Hopf algebra (with unit and co-unit) $R_{0} / k$, we wish to create an SDC describing flat deformations $R_{A} / A$ of $R_{0}$ such that $R_{A} \otimes_{A} k=R_{0}$, modulo infinitesimal isomorphisms.

The structures are:

$$
\Sigma^{+}=\{\text {Algebra }\}, \quad \Sigma^{-}=\{\text {Co-Algebra }\},
$$

over the category of flat $A$-modules.

This gives the following commutative diagram of monadic and comonadic adjunctions:

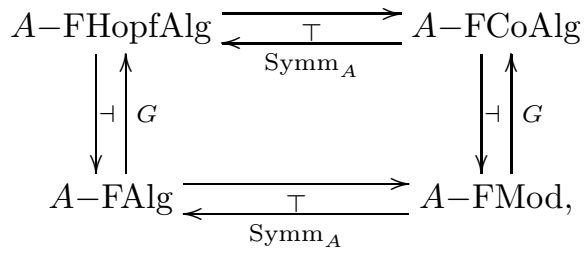

where $A$-FHopfAlg is the category of flat Hopf algebras over $A, A-$ FCoAlg is the category of flat co-associative co-algebras with co-unit and co-inverse over $A$, and $G$ is the free co-algebra functor of Section 1.3.2. Since we cannot describe $G$ explicitly, we use the alternative form of the axioms involving $\operatorname{Symm}_{A}$ instead, when verifying the conditions of Theorem 2.2.

We thus obtain the SDC

$$
E^{n}=\operatorname{Hom}_{\Lambda}\left(\left(\operatorname{Symm}_{\Lambda}\right)^{n} M, G^{n} M\right)_{\alpha^{n} \circ \varepsilon^{n},}
$$

where $M$ is the flat $\mu$-adic $\Lambda$-module (unique up to isomorphism) lifting the $k$-vector space $R_{0}$, and $\alpha^{n}, \varepsilon^{n}$ are the canonical maps

$$
\varepsilon^{n}:\left(\mathrm{Symm}_{k}\right)^{n} R_{0} \rightarrow R_{0}, \quad \alpha^{n}: R_{0} \rightarrow G^{n} R_{0}
$$

associated to $R_{0}$.

3.2. Deformations of schemes. Given a flat $\mu$-adic system of schemes $S_{n} /$ $\operatorname{Spec} \Lambda_{n}$, let $\mathfrak{S}=\underline{\lim } S_{n}$ and $S_{A}=\mathfrak{S} \times \operatorname{Spf} \Lambda$ Spec $A$. Given a scheme $X_{0} / S_{0}$, our deformation functor consists of schemes $X_{A} / S_{A}$, with $X_{A} \rightarrow \operatorname{Spec} A$ flat, such that $X_{A} \times_{\operatorname{Spec} A} \operatorname{Spec} k=X_{0}$, modulo infinitesimal isomorphisms (isomorphisms which pull back to the identity on $X_{0}$ ):

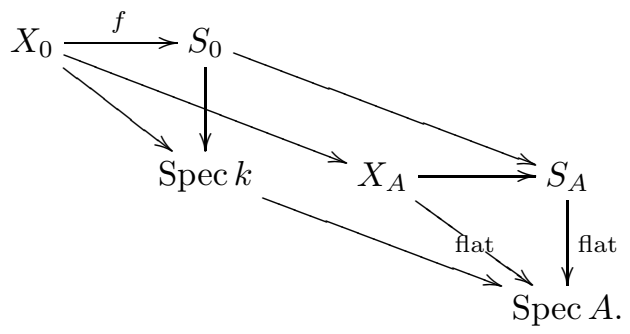


Since the topological space $\left|X_{0}\right|$ underlying $X_{0}$ does not deform, this is just a question of deforming the sheaf $\mathscr{O}_{X_{0}}$ of algebras. Explicitly, it suffices to deform $\mathscr{O}_{X_{0}}$ as an $\mathscr{O}_{S_{A}}$-algebra, flat over $A$.

In the notation of Section 2, we have the set of structures

$$
\Sigma^{+}=\left\{\text {algebra, } \mathscr{O}_{S_{A}} \text {-module }\right\}, \quad \Sigma^{-}=\left\{X_{0} \text {-sheaf }\right\},
$$

over the category of sheaves of flat $A$-modules on $X_{0}^{\prime}$, where $X_{0}^{\prime}$ is defined as in Section 1.3.1. To these structures correspond the monadic and comonadic adjunctions

$$
\left\{\operatorname{Symm}_{A} \dashv U, \mathscr{O}_{\mathfrak{S}} \otimes_{\Lambda} \dashv U\right\}, \quad\left\{u_{*} \vdash u^{-1}\right\},
$$

where $U$ denotes the relevant forgetful functor.

This yields the following diagram of Cat-valued functors:

$$
\begin{aligned}
& \begin{aligned}
\mathscr{O}_{S_{A}} \downarrow\left(A-\mathrm{FAlg}\left(X_{0}\right)\right) \underset{\mathscr{O}_{\mathfrak{S} \otimes_{\Lambda} \operatorname{Symm}_{A}}}{\frac{T}{\longrightarrow}} & A-\operatorname{FMod}\left(X_{0}\right) \\
u^{-1}|\dashv| u_{*} & u^{-1}|\dashv| u_{*}
\end{aligned} \\
& \mathscr{O}_{S_{A}} \downarrow\left(A-\mathrm{FAlg}\left(X_{0}^{\prime}\right)\right) \underset{\mathscr{O}_{\mathfrak{S}} \otimes_{\Lambda} \operatorname{Symm}_{A}}{\underset{T}{\rightleftarrows}} A-\operatorname{FMod}\left(X_{0}^{\prime}\right),
\end{aligned}
$$

where we write $\mathscr{O}_{\mathfrak{S}}$ for the sheaf $f^{-1} \mathscr{O}_{\mathfrak{S}}$ (resp. $\left.u^{-1} f^{-1} \mathscr{O}_{\mathfrak{S}}\right)$ on $X_{0}$ (resp. $X_{0}^{\prime}$ ), and $\mathscr{O}_{S_{A}} \downarrow(A-$ FAlg $)$ consists of those $\mathscr{O}_{S_{A}}$-algebras which are flat over $A$. The only non-trivial commutativity condition is the observation that pull-backs commute with tensor operations.

Hence, by Theorem 2.2, deformations are described by the SDC

$$
E^{n}=\operatorname{Hom}_{\Lambda}\left(\left(\mathscr{O}_{\mathfrak{S}} \otimes_{\Lambda} \operatorname{Symm}_{\Lambda}\right)^{n} \mathscr{M},\left(u^{-1} u_{*}\right)^{n} \mathscr{M}\right)_{u^{-1}\left(\alpha^{n} \circ \varepsilon^{n}\right)},
$$

where $\mathscr{M}$ is a lift of the sheaf $u^{-1} \mathscr{O}_{X_{0}}$ of vector spaces on $X_{0}^{\prime}$ to a sheaf of flat $\mu$-adic $\Lambda$-modules, and $\alpha^{n}, \varepsilon^{n}$ are the canonical maps $\alpha_{\mathscr{O}_{X_{0}}}^{n}, \varepsilon_{\mathscr{O}_{X_{0}}}^{n}$ in Theorem 2.2, given by the adjunctions.

Remark 3.1. Observe that the description above allows us to construct an SDC governing deformations of a sheaf of algebras on any site with enough points. This is more general than the problem in [2] for which a DGLA was constructed.

Define a sheaf of SDCs on $X$ by

$$
\mathscr{E}^{n}=u_{*} \mathscr{H} o m_{\Lambda}\left(\left(\mathscr{O}_{\mathfrak{S}} \otimes_{\Lambda} \operatorname{Symm}_{\Lambda}\right)^{n} \mathscr{M},\left(u^{-1} u_{*}\right)^{n} \mathscr{M}\right)_{u^{-1}\left(\alpha^{n} \circ \varepsilon^{m}\right)} .
$$

Combining the observations in $[6, \S \S 1.2 .1,1.2 .3]$, we see that this has tangent space

$$
\begin{aligned}
\mathrm{C}^{n}(\mathscr{E}) & =u_{*}{\mathscr{D} e \mathscr{O}_{S_{0}}}\left(\left(\mathscr{O}_{S_{0}} \otimes_{k} \operatorname{Symm}_{k}\right)^{n+1} u^{-1} \mathscr{O}_{X_{0}},\left(u^{-1} u_{*}\right)^{n} u^{-1} \mathscr{O}_{X_{0}}\right) \\
& \cong \operatorname{Der}_{\mathscr{O}_{S_{0}}}\left(\left(\mathscr{O}_{S_{0}} \otimes_{k} \operatorname{Symm}_{k}\right)^{n+1} \mathscr{O}_{X_{0}},\left(u_{*} u^{-1}\right)^{n+1} \mathscr{O}_{X_{0}}\right) \\
& \cong \mathscr{H}_{o m_{\mathscr{O}_{X_{0}}}}\left(\mathbf{L}_{n}^{X_{0} / S_{0}}, \mathscr{C}^{n}\left(\mathscr{O}_{X_{0}}\right)\right),
\end{aligned}
$$

where $\mathbf{L}_{\bullet}^{X_{0} / S_{0}}$ is the standard form of Illusie's cotangent complex, as described in [3], and $\mathscr{C}^{n}$ denotes the Godement resolution.

Since $C^{n}(\mathscr{E})$ has the structure of a diagonal complex of a bicosimplicial complex, it follows from the Eilenberg-Zilber Theorem that it is quasi-isomorphic to the total complex of the double complex

$$
\mathscr{H}_{0 m_{O_{X_{0}}}}\left(\mathbf{L}_{\bullet}^{X_{0} / S_{0}}, \mathscr{C}^{\bullet}\left(\mathscr{O}_{X_{0}}\right)\right)
$$


Therefore the cohomology of our SDC is

$$
\mathrm{H}^{i}\left(\mathrm{C}^{\bullet}(E)\right)=\mathrm{H}^{i}\left(\Gamma\left(X_{0}, \mathrm{C}^{\bullet}(\mathscr{E})\right)\right) \cong \mathbb{E x t}_{\mathscr{O}_{X_{0}}}^{i}\left(\mathbf{L}_{\bullet}^{X_{0} / S_{0}}, \mathscr{O}_{X_{0}}\right),
$$

which is André-Quillen hypercohomology, the second isomorphism following because $\mathbf{L}_{\bullet}^{X_{0} / S_{0}}$ is locally projective as an $\mathscr{O}_{X_{0}}$-module and $\mathscr{C} \bullet\left(\mathscr{O}_{X_{0}}\right)$ is flabby.

3.2.1. Separated Noetherian schemes. If $X_{0}$ is separated and Noetherian, then we may replace Godement resolutions by Čech resolutions. Take an open affine cover $\left(X_{\alpha}\right)_{\alpha \in I}$ of $X_{0}$, and set $\check{X}:=\coprod_{\alpha \in I} X_{\alpha}$. We then have a diagram

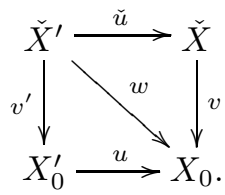

Since $v^{-1} \mathscr{O}_{X_{0}}=\mathscr{O}_{\check{X}}$ is a quasi-coherent sheaf on $\check{X}, \operatorname{Ext}^{i}\left(\mathscr{O}_{\check{X}}, \mathscr{O}_{\check{X}}\right)=0$ for all $i>0$, so deformations of the sheaf $\mathscr{O}_{\tilde{X}}$ of $k$-vector spaces on $\dot{X}$ are unobstructed and we may lift $v^{-1} \mathscr{O}_{X_{0}}$ to some sheaf $\mathscr{N}$ of flat $\mu$-adic $\Lambda$-modules on $\check{X}$, unique up to non-unique isomorphism. There must also be (non-canonical) isomorphisms $\check{u}^{-1} \mathscr{N} \cong v^{\prime-1} \mathscr{M}$. If we define $\check{E}^{\bullet}$ by

$$
\check{E}^{n}:=\operatorname{Hom}_{\Lambda}\left(\left(\mathscr{O}_{\mathfrak{S}} \otimes_{\Lambda} \operatorname{Symm}_{\Lambda}\right)^{n} \mathscr{N},\left(v^{-1} v_{*}\right)^{n} \mathscr{N}\right)_{v^{-1}\left(\alpha^{n} \circ \varepsilon^{n}\right)},
$$

and $F^{\bullet}$ similarly, replacing $v$ by $w$, then we have morphisms of SDCs

$$
E^{\bullet} \stackrel{\gamma_{v^{\prime}}}{\longrightarrow} F^{\bullet} \stackrel{\gamma_{\dot{u}}^{\bullet}}{\longleftarrow} \check{E}^{\bullet} \text {. }
$$

To see that these are quasi-isomorphisms, let $\mathscr{T}^{\bullet}:=\mathscr{H}_{0} m_{\mathscr{O}_{X_{0}}}\left(\mathbf{L}_{\bullet}^{X_{0} / S_{0}}, \mathscr{O}_{X_{0}}\right)$, which is a complex of quasi-coherent sheaves on $X_{0}$, and observe that the induced maps on cohomology are

$$
\mathbb{H}^{i}\left(X_{0}, \mathscr{T}^{\bullet}\right) \rightarrow \mathbb{H}^{i}\left(X_{0}, \mathscr{T}^{\bullet}\right) \leftarrow \check{\mathbb{H}}^{i}\left(X_{0}, \mathscr{T}^{\bullet}\right)
$$

The final map is an isomorphism since $X_{0}$ is separated and Noetherian, so Cech cohomology agrees with sheaf cohomology.

3.2.2. Smooth schemes. If $X_{0} / S_{0}$ is smooth, then the cohomology groups are just $\mathrm{H}^{i}\left(X_{0}, \mathscr{T}_{X_{0} / S_{0}}\right)$, the cohomology of the tangent sheaf. If $X_{0}$ is also separated and Noetherian (for instance if $S_{0}$ is so), then we may lift $\check{X}$ to some smooth $\mu$-adic formal scheme $\check{\mathfrak{X}}$ over $\mathfrak{S}$.

Consider the diagram

$$
\mathscr{O}_{S_{A}} \downarrow(A-\mathrm{FAlg}(\check{X})) \underset{v^{*}}{\stackrel{v_{*}}{\underset{\mathrm{T}}{\longrightarrow}}} \mathscr{O}_{S_{A}} \downarrow\left(A-\mathrm{FAlg}\left(X_{0}\right)\right) ;
$$

although the former category does not have uniformly trivial deformation theory, all the morphisms in it which we encounter do, which gives us an SDC and canonical maps

$$
\operatorname{Hom}_{\mathscr{O}_{\mathfrak{S}}-\operatorname{Alg}}\left(\mathscr{O}_{\check{\mathfrak{X}}},\left(v^{-1} v_{*}\right)^{n} \mathscr{O}_{\check{\mathfrak{X}}}\right)_{v^{-1}\left(\alpha^{n}\right)} \stackrel{\left(\varepsilon^{n}\right)^{*}}{\longrightarrow} \check{E}^{n},
$$

which give a quasi-isomorphism of SDCs.

Explicitly, we may write our SDC as

$$
E^{n}=\prod_{\alpha_{0}, \ldots, \alpha_{n} \in I} \Gamma\left(X_{\alpha_{0}, \ldots, \alpha_{n}}, \mathscr{H}_{0} m_{\mathscr{O}_{\mathfrak{S}}-\operatorname{Alg}}\left(\mathscr{O}_{\mathfrak{X}_{\alpha_{n}}}, \mathscr{O}_{\mathfrak{X}_{\alpha_{0}}}\right)_{\mathrm{id}}\right),
$$


where $X_{\alpha_{0}, \ldots, \alpha_{n}}=\bigcap_{i=1}^{n} X_{\alpha_{i}}$, and $\check{\mathfrak{X}}=\coprod \mathfrak{X}_{\alpha}$. The product is given by

$$
(\phi * \psi)_{\alpha_{0}, \ldots, \alpha_{m+n}}=\phi_{\alpha_{0}, \ldots, \alpha_{m}} \circ \psi_{\alpha_{m}, \ldots, \alpha_{m+n}},
$$

and operations are

$$
\begin{aligned}
\left(\partial^{i} \phi\right)_{\alpha_{0}, \ldots, \alpha_{n+1}} & =\phi_{\alpha_{0}, \ldots, \alpha_{i-1}, \alpha_{i+1}, \ldots, \alpha_{n+1}} \\
\left(\sigma^{i} \phi\right)_{\alpha_{0}, \ldots, \alpha_{n-1}} & =\phi_{\alpha_{0}, \ldots, \alpha_{i}, \alpha_{i}, \ldots, \alpha_{n-1}}
\end{aligned}
$$

3.2.3. Deformations of a subscheme. Although the standard application of the previous section would be to set $S_{n}=\operatorname{Spec} \Lambda_{n}$, it applies to a far wider class of problems. In particular this applies to deformations of a subscheme $X_{0} \hookrightarrow S_{0}$, since a flat deformation of a subscheme as an $S_{A}$-scheme will be a subscheme. If $X_{0} \hookrightarrow S_{0}$ is a regular embedding (e.g., if $X_{0}$ and $S_{0}$ are both smooth over Spec $k$ ), then the cohomology groups will be

$$
\mathrm{H}^{i}\left(\mathrm{C}^{\bullet}(E)\right)=\mathrm{H}^{i+1}\left(X_{0}, \mathscr{N}_{X_{0} / S_{0}}\right),
$$

where $\mathscr{N}_{X_{0} / S_{0}}$ is the normal sheaf.

If $X_{0}$ and $S_{0}$ are both smooth over Spec $k$, we can simplify the SDC of Section 3.2.1 still further. Consider the diagram

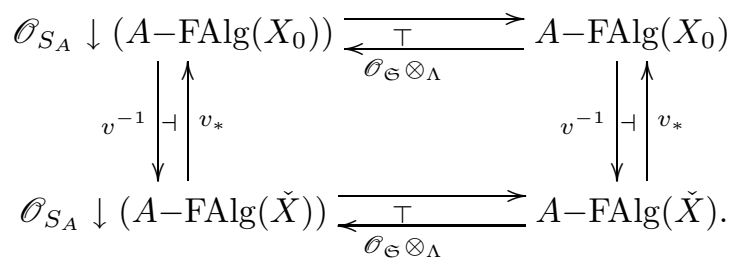

Although the last category does not have uniformly trivial deformation theory, all the morphisms in it which we encounter do. If we let $\left(f^{\sharp}\right)^{\otimes n}$ denote the canonical ring homomorphism $\mathscr{O}_{\mathfrak{S}}^{\otimes n} \otimes \mathscr{O}_{\hat{\mathfrak{x}}} \rightarrow \mathscr{O}_{\hat{\mathfrak{x}}}$, then there is an SDC and a quasiisomorphism

$$
\operatorname{Hom}_{\Lambda-\operatorname{Alg}}\left(\mathscr{O}_{\mathfrak{S}}^{\otimes n} \otimes \mathscr{O}_{\hat{\mathfrak{X}}},\left(v^{-1} v_{*}\right)^{n} \mathscr{O}_{\hat{\mathfrak{X}}}\right)_{v^{-1} \alpha^{n} \circ\left(f^{\sharp}\right) \otimes n} \rightarrow \check{E}^{n} .
$$

The map is defined by composing the maps $\operatorname{Symm}_{\Lambda}\left(\mathscr{O}_{\mathfrak{S}}^{\otimes n} \otimes \mathscr{O}_{\hat{\mathfrak{X}}}\right) \rightarrow \mathscr{O}_{\mathfrak{S}}^{\otimes n} \otimes \mathscr{O}_{\hat{\mathfrak{X}}}$, arising from the natural ring structure on the tensor product.

Explicitly, we may write our SDC as

$$
E^{n}=\prod_{\alpha_{0}, \ldots, \alpha_{n} \in I} \Gamma\left(X_{\alpha_{0}, \ldots, \alpha_{n}}, \mathscr{H}_{0 m_{\Lambda-A l g}}\left(\mathscr{O}_{\mathfrak{S}}^{\otimes n} \otimes \mathscr{O}_{\mathfrak{X}_{\alpha_{n}}}, \mathscr{O}_{\mathfrak{X}_{\alpha_{0}}}\right)_{\left(f^{\sharp}\right) \otimes n}\right) .
$$

The product is given by

$$
\begin{gathered}
(\phi * \psi)_{\alpha_{0}, \ldots, \alpha_{m+n}}\left(r_{1} \otimes \ldots \otimes r_{m+n} \otimes a\right) \\
=\phi_{\alpha_{0}, \ldots, \alpha_{m}}\left(r_{1} \otimes \ldots \otimes r_{m} \otimes \psi_{\alpha_{m}, \ldots, \alpha_{m+n}}\left(r_{m+1} \otimes \ldots \otimes r_{m+n} \otimes a\right)\right),
\end{gathered}
$$

and the operations are

$$
\begin{aligned}
& \left(\partial^{i} \phi\right)_{\alpha_{0}, \ldots, \alpha_{n+1}}\left(r_{1} \otimes \ldots \otimes r_{n+1} \otimes a\right) \\
= & \phi_{\alpha_{0}, \ldots, \alpha_{i-1}, \alpha_{i+1}, \ldots, \alpha_{n+1}}\left(r_{1} \otimes \ldots \otimes r_{i-1} \otimes\left(r_{i} r_{i+1}\right) \otimes r_{i+2} \ldots \otimes r_{n+1} \otimes a\right), \\
& \left(\sigma^{i} \phi\right)_{\alpha_{0}, \ldots, \alpha_{n-1}}\left(r_{1} \otimes \ldots \otimes r_{n-1} \otimes a\right) \\
= & \phi_{\alpha_{0}, \ldots, \alpha_{i}, \alpha_{i}, \ldots, \alpha_{n-1}}\left(r_{1} \otimes \ldots \otimes r_{i} \otimes 1 \otimes r_{i+1} \ldots \otimes r_{n-1} \otimes a\right) .
\end{aligned}
$$

To see that the map is a quasi-isomorphism, observe that the Eilenberg-Zilber Theorem allows us to regard the cohomology of $E$ as the hypercohomology of the 
complex $\mathscr{T}_{X_{0} / k} \rightarrow f^{*} \mathscr{T}_{S_{0} / k}[-1]$, which is quasi-isomorphic to the tangent complex of the morphism $X_{0} \rightarrow S_{0}$. Note that this SDC works for any morphism of smooth schemes, not only for embeddings.

3.3. Constrained deformations of schemes. Given a morphism of flat $\mu$-adic schemes $\mathfrak{Z} \stackrel{h}{\longrightarrow} \mathfrak{S}$ over $\operatorname{Spf} \Lambda$, and a diagram

$$
Z_{0} \stackrel{g}{\rightarrow} X_{0} \stackrel{f}{\rightarrow} S_{0}
$$

over $k$, with $f g=h$, our deformation functor consists of those diagrams

$$
Z_{A} \stackrel{g_{A}}{\longrightarrow} X_{A} \stackrel{f_{A}}{\longrightarrow} S_{A}
$$

with $X_{A} \rightarrow \operatorname{Spec} A$ flat and $f_{A} \circ g_{A}=h$, which pull back along $\operatorname{Spec} k \rightarrow \operatorname{Spec} A$ to our original diagram. Here, $Z_{A}$ is the scheme $\mathfrak{Z} \times \operatorname{Spf} \Lambda \operatorname{Spec} A$, and $Z_{0}=Z_{k}$. An example of such a deformation problem would be deformations of a subscheme, constrained to pass through a fixed set of points.

Since the topological space $\left|X_{0}\right|$ underlying $X_{0}$ does not deform, it suffices to deform $\mathscr{O}_{X_{0}}$ as an $\mathscr{O}_{S_{A}}$-algebra with an $\mathscr{O}_{Z_{A}}$-augmentation, flat over $A$.

In the notation of Section 2, we have the set of structures

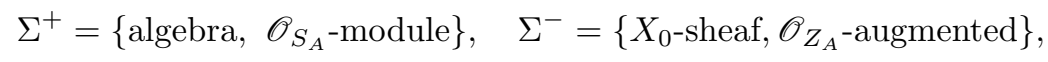

over the category of sheaves of flat $A$-modules on $X_{0}^{\prime}$, where $X_{0}^{\prime}$ is defined as in Section 1.3.1.

To these structures correspond the monadic and comonadic adjunctions

$$
\left\{\operatorname{Symm}_{A} \dashv U, \mathscr{O}_{\mathfrak{S}} \otimes_{\Lambda} \dashv U\right\}, \quad\left\{u_{*} \vdash u^{-1}, \mathscr{O}_{\mathfrak{Z}} \times \vdash V\right\},
$$

where $U, V$ denote the relevant forgetful functors.

This yields the following diagram of Cat-valued functors:

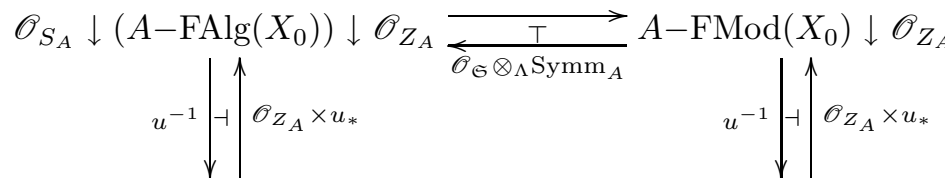

$$
\begin{aligned}
& \mathscr{O}_{S_{A}} \downarrow\left(A-\mathrm{FAlg}\left(X_{0}^{\prime}\right)\right) \underset{\mathscr{O}_{\mathfrak{S}} \otimes_{\Lambda} \operatorname{Symm}_{A}}{\stackrel{F}{\rightleftarrows}} A-\mathrm{FMod}\left(X_{0}^{\prime}\right),
\end{aligned}
$$

where we write $\mathscr{O}_{\mathfrak{S}}$ for the sheaf $f^{-1} \mathscr{O}_{\mathfrak{S}}\left(\right.$ resp. $\left.u^{-1} f^{-1} \mathscr{O}_{\mathfrak{S}}\right)$, and $\mathscr{O}_{\mathfrak{Z}}$ for the sheaf $g_{*} \mathscr{O}_{\mathfrak{Z}}\left(\right.$ resp. $\left.u^{-1} g_{*} \mathscr{O}_{\mathfrak{Z}}\right)$ on $X_{0}$ (resp. $X_{0}^{\prime}$ ). The category $\mathscr{O}_{S_{A}} \downarrow(A-$ FAlg $) \downarrow \mathscr{O}_{Z_{A}}$ consists of those $\mathscr{O}_{S_{A}}$-algebras which are flat over $A$ and equipped with an augmentation to $\mathscr{O}_{Z_{A}}$, compatible with $h^{\sharp}$. The only non-trivial commutativity condition is the observation that pull-backs commute with tensor operations.

By Theorem 2.2, this deformation problem is governed by the SDC

$$
E^{n}=\operatorname{Hom}_{\Lambda}\left(\left(\mathscr{O}_{\mathfrak{S}} \otimes_{\Lambda} \operatorname{Symm}_{\Lambda}\right)^{n} \mathscr{M},\left(\mathscr{O}_{\mathfrak{Z}} \times u^{-1} u_{*}\right)^{n} \mathscr{M}\right)_{u^{-1}\left(\alpha^{n} \circ \varepsilon^{n}\right)},
$$

where $\mathscr{M}$ is a lift of the sheaf $u^{-1} \mathscr{O}_{X_{0}}$ of vector spaces on $X_{0}^{\prime}$ to a sheaf of flat $\mu$-adic $\Lambda$-modules, and $\alpha^{n}, \varepsilon^{n}$ are the canonical maps $\alpha_{\mathscr{O}_{X_{0}}}^{n}, \varepsilon_{\mathscr{O}_{X_{0}}}^{n}$ in Theorem 2.2 given by the adjunctions. The cohomology is

$$
\mathbb{E x t}_{\mathscr{O}_{X_{0}}}^{i}\left(\mathbf{L}_{\bullet}^{X_{0} / S_{0}}, \mathscr{O}_{X_{0}} \stackrel{g^{\sharp}}{\rightarrow} g_{*} \mathscr{O}_{Z_{0}}[-1]\right) .
$$


If $X_{0}$ and $S_{0}$ are both smooth, we may consider the diagram

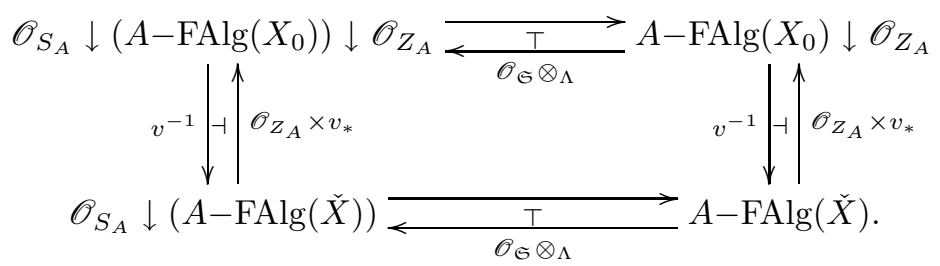

All the morphisms which we encounter in the last category have uniformly trivial deformation theory, allowing us to replace this SDC by

$$
E^{n}=\prod_{\alpha_{0}, \ldots, \alpha_{n} \in I} \Gamma\left(X_{\alpha_{0}, \ldots, \alpha_{n}}, \mathscr{H}_{0 m_{\Lambda-A l g}}\left(\mathscr{O}_{\mathfrak{S}}^{\otimes n} \otimes \mathscr{O}_{\mathfrak{X}_{\alpha_{n}}}, \mathscr{O}_{\mathfrak{X}_{\alpha_{0}}} \times \mathscr{O}_{\mathfrak{Z}}^{n}\right)_{\left(g^{\sharp}\right)^{n} \circ\left(f^{\sharp}\right) \otimes n}\right),
$$

since cohomology in this case is hypercohomology of the complex

$$
\mathscr{T}_{X_{0} / k} \rightarrow\left(f^{*} \mathscr{T}_{S_{0} / k} \oplus \mathscr{T}_{X_{0} / k} \otimes_{\mathscr{O}_{X_{0}}} g_{*} \mathscr{O}_{Z_{0}}\right)[-1] \rightarrow f^{*} \mathscr{T}_{S_{0} / k} \otimes_{\mathscr{O}_{X_{0}}} g_{*} \mathscr{O}_{Z_{0}}[-2]
$$

3.4. Deformations of group schemes. Given a group scheme $G_{0} / k$, we consider deformations

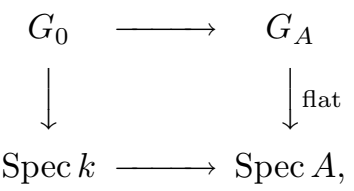

where $G_{A}$ is a group scheme over $A$ with $G_{A} \otimes_{A} k=G_{0}$.

Since the topological space $\left|G_{0}\right|$ does not deform, we need only consider deformations of $\mathscr{O}_{G_{0}}$ as a sheaf of Hopf algebras. The structures are:

$$
\Sigma^{+}=\{\text {algebra }\}, \quad \Sigma^{-}=\left\{G_{0^{-}} \text {sheaf, co-algebra }\right\},
$$

over the category of sheaves of flat $A$-modules on $G_{0}^{\prime}$.

This gives the following commutative diagram of monadic and comonadic adjunctions:

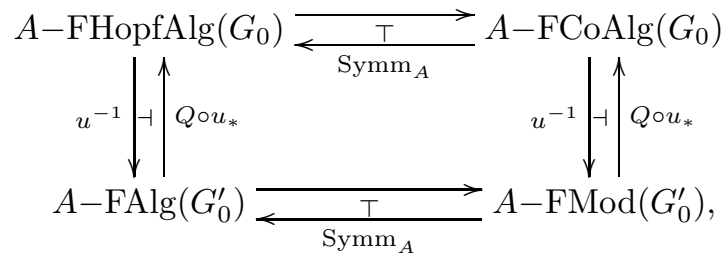

where $A$-FHopfAlg is the category of flat Hopf algebras over $A, A$-FCoAlg is the category of flat co-associative co-algebras with co-unit and co-inverse over $A$, and $Q$ is the free co-algebra functor of Section 1.3.2. We thus obtain the SDC

$$
E^{n}=\operatorname{Hom}_{\Lambda}\left(\left(\operatorname{Symm}_{\Lambda}\right)^{n} \mathscr{M},\left(u_{*} \circ Q \circ u^{-1}\right)^{n} \mathscr{M}\right)_{u^{-1}\left(\alpha^{n} \circ \varepsilon^{n}\right)},
$$

where $\mathscr{M}$ is the lift (unique up to isomorphism) of the sheaf $u^{-1} \mathscr{O}_{G_{0}}$ of $k$-vector spaces to a flat $\mu$-adic sheaf on $G^{\prime}$, with $\alpha^{n}, \varepsilon^{n}$ the canonical maps

$$
\varepsilon^{n}:\left(\operatorname{Symm}_{k}\right)^{n} \mathscr{O}_{G_{0}} \rightarrow \mathscr{O}_{G_{0}}, \quad \alpha^{n}: \mathscr{O}_{G_{0}} \rightarrow\left(u_{*} \circ Q \circ u^{-1}\right)^{n} \mathscr{O}_{G_{0}}
$$

associated to $\mathscr{O}_{G_{0}}$. 
4. Equivalence Between SDCs And DGLAs in CharaCteristic Zero

Throughout this section, we will assume that $\Lambda=k$, a field of characteristic zero. We will also assume that all DGLAs are in non-negative degrees, i.e. $L^{<0}=0$.

4.1. Review of DGLAs. The results of this section can all be found in [5].

Definition 4.1. A DGLA over a field $k$ of characteristic 0 is a graded vector space $L=\bigoplus_{i \in \mathbb{N}_{0}} L^{i}$ over $k$, equipped with operators [,] : $L \times L \rightarrow L$ bilinear and $d: L \rightarrow L$ linear, satisfying:

(1) $\left[L^{i}, L^{j}\right] \subset L^{i+j}$

(2) $[a, b]+(-1)^{\bar{a} \bar{b}}[b, a]=0$,

(3) $(-1)^{\bar{c} \bar{a}}[a,[b, c]]+(-1)^{\bar{a} \bar{b}}[b,[c, a]]+(-1)^{\bar{b} \bar{c}}[c,[a, b]]=0$,

(4) $d\left(L^{i}\right) \subset L^{i+1}$,

(5) $d \circ d=0$,

(6) $d[a, b]=[d a, b]+(-1)^{\bar{a}}[a, d b]$.

Here $\bar{a}$ denotes the degree of $a, \bmod 2$, for $a$ homogeneous.

Fix a DGLA $L$.

Definition 4.2. The Maurer-Cartan functor $\mathrm{MC}_{L}: \mathcal{C}_{k} \rightarrow$ Set is defined by

$$
\mathrm{MC}_{L}(A)=\left\{x \in L^{1} \otimes \mathfrak{m}_{A} \mid d x+\frac{1}{2}[x, x]=0\right\} .
$$

Observe that for $\omega \in L^{1} \otimes \mathfrak{m}_{A}$,

$$
d \omega+\frac{1}{2}[\omega, \omega]=0 \Rightarrow\left(d+\operatorname{ad}_{\omega}\right) \circ\left(d+\operatorname{ad}_{\omega}\right)=0,
$$

so $\left(L \otimes A,[],, d+\operatorname{ad}_{\omega}\right)$ is a DGLA over $A$.

Definition 4.3. Define the gauge functor $\mathrm{G}_{L}: \mathcal{C}_{k} \rightarrow \operatorname{Grp}$ by $\mathrm{G}_{L}(A)=$ $\exp \left(L^{0} \otimes \mathfrak{m}_{A}\right)$.

We may define another DGLA, $L_{d}$, by

$$
L_{d}^{i}=\left\{\begin{array}{cc}
L^{1} \oplus k d & i=1 \\
L^{i} & i \neq 1
\end{array}\right.
$$

with

$$
d_{d}(d)=0, \quad[d, d]=0, \quad[d, a]_{d}=d a, \quad \forall a \in L .
$$

Lemma 4.4. $\exp \left(L^{0} \otimes \mathfrak{m}_{A}\right)$ commutes with [,] when acting on $L_{d} \otimes A$ via the adjoint action.

Corollary 4.5. Since $\exp \left(L^{0} \otimes \mathfrak{m}_{A}\right)$ preserves $L^{1} \otimes \mathfrak{m}_{A}+d \subset L_{d} \otimes A$ under the adjoint action, and

$$
x \in \mathrm{MC}_{L}(A) \Leftrightarrow[x+d, x+d]=0,
$$

the adjoint action of $\exp \left(L^{0} \otimes \mathfrak{m}_{A}\right)$ on $L^{1} \otimes A+d$ induces an action of $\mathrm{G}_{L}(A)$ on $\mathrm{MC}_{L}(A)$, which we will call the gauge action.

Definition 4.6. $\operatorname{Def}_{L}=\mathrm{MC}_{L} / \mathrm{G}_{L}$, the quotient being given by the gauge action $\alpha(x)=\operatorname{ad}_{\alpha}(x+d)-d$. Observe that $\mathrm{G}_{L}$ is homogeneous. Define the deformation groupoid $\mathfrak{D} \mathfrak{e} \mathfrak{f}_{L}$ to have objects $\mathrm{MC}_{L}$, and morphisms given by $\mathrm{G}_{L}$. 
Theorem 4.7. $\operatorname{Def}_{L}$ is a deformation functor, $t_{\text {Def }_{L}} \cong \mathrm{H}^{1}(L)$, and $\mathrm{H}^{2}(L)$ is a complete obstruction space for $\operatorname{Def}_{L}$. For $x, y$ objects in $\mathfrak{D e f}_{L}(A)$, the group-valued functor $\operatorname{Iso}(x, y): \mathcal{C}_{A} \rightarrow \operatorname{Grp}$ is homogeneous, with tangent space $\mathrm{H}^{0}(L)$ and complete obstruction space $\mathrm{H}^{1}(L)$.

Theorem 4.8. If $\phi: L \rightarrow M$ is a morphism of DGLAs, and

$$
\mathrm{H}^{i}(\phi): \mathrm{H}^{i}(L) \rightarrow \mathrm{H}^{i}(M)
$$

are the induced maps on cohomology, then:

(1) If $\mathrm{H}^{1}(\phi)$ is bijective, and $\mathrm{H}^{2}(\phi)$ injective, then $\operatorname{Def}_{L} \rightarrow \operatorname{Def}_{M}$ is étale.

(2) If also $\mathrm{H}^{0}(\phi)$ is surjective, then $\operatorname{Def}_{L} \rightarrow \operatorname{Def}_{M}$ is an isomorphism.

(3) Provided condition (1) holds, $\mathfrak{D e f}_{L} \rightarrow \mathfrak{D e f}_{M}$ is an equivalence of functors of groupoids if and only if $\mathrm{H}^{0}(\phi)$ is an isomorphism.

Proof. See [5, Theorem 3.1].

Thus, in particular, a quasi-isomorphism of DGLAs gives an isomorphism of deformation functors and deformation groupoids.

Finally, we make an observation which does not appear in [5]. Define $\mathcal{C}_{k}^{\mathbb{N}_{0}}$ to be the category of nilpotent local Noetherian graded (super-commutative) $k$-algebras (in non-negative degrees) with residue field $k$ (concentrated in degree 0).

Lemma 4.9. Given a pro-representable (in the sense of [1]) functor $G: \mathcal{C}_{k}^{\mathbb{N}_{0}} \rightarrow$ Grp, with tangent space the GLA $L^{*}$, there is a canonical isomorphism

$$
G\left(A_{*}\right) \cong \exp (L)\left(A_{*}\right):=\exp \left(\bigoplus_{n} L^{n} \otimes\left(\mathfrak{m}_{A}\right)_{n}\right) .
$$

In particular, this implies that $G$ must be smooth.

Proof. Let $G$ be pro-represented by $\mathcal{T}_{*}$, the product corresponding to a map $\rho$ : $\mathcal{T}_{*} \rightarrow \mathcal{T}_{*} \hat{\otimes} \mathcal{T}_{*}$. Then

$$
G\left(A_{*}\right)=\operatorname{Hom}_{k-\operatorname{Alg}}\left(\mathcal{T}_{*}, A_{*}\right), \quad \bigoplus_{n} L^{n} \otimes\left(\mathfrak{m}_{A}\right)_{n}=\operatorname{Der}_{k}\left(\mathcal{T}_{*}, A_{*}\right) .
$$

We can embed both of these into $\operatorname{Hom}_{k}\left(\mathcal{T}_{*}, A_{*}\right)$, on which we define the associative product $f * g=(f \otimes g) \circ \rho$. Now the maps

$$
\begin{aligned}
G\left(A_{*}\right) & \cong \bigoplus_{n} L^{n} \otimes\left(\mathfrak{m}_{A}\right)_{n} \\
g & \mapsto \sum_{n \geq 1}(-1)^{n-1} \frac{(g-e)^{* n}}{n}, \\
\sum_{n \geq 0} \frac{l^{* n}}{n !} & \leftrightarrow l
\end{aligned}
$$

where $e$ is the identity map $\mathcal{T}_{*} \rightarrow k$, give us our isomorphism.

4.2. DGLA $\rightsquigarrow$ SDC. Given a DGLA $L$, first form the denormalised cosimplicial complex

$$
\left(N^{-1} L\right)^{n}:=\bigoplus_{\substack{m+s=n \\ 1 \leq j_{1}<\ldots<j_{s} \leq n}} \partial^{j_{s}} \ldots \partial^{j_{1}} L^{m}
$$


where we define the $\partial^{j}$ and $\sigma^{i}$ using the simplicial identities, subject to the conditions that for all $v \in L^{n}, \sigma^{i} v=0$ and $d v=\sum_{i=0}^{n+1}(-1)^{i} \partial^{i} v$.

Given $v, w \in\left(N^{-1} L\right)^{n}$ such that $\sigma^{i} v \otimes \sigma^{i} w=0$ for all $i$, define

$$
\llbracket v, w \rrbracket:=\sum_{\substack{p+q=n \\(\mu, \nu) \in \operatorname{Sh}(p, q)}}(-1)^{(\mu, \nu)}\left[\sigma^{\nu_{1}} \ldots \sigma^{\nu_{q}} v, \sigma^{\mu_{1}} \ldots \sigma^{\mu_{p}} w\right] \in L^{n},
$$

with $\operatorname{Sh}(p, q)$ denoting the set of $(p, q)$ shuffle permutations, i.e. permutations

$$
\left(\mu_{1}, \ldots, \mu_{p}, \nu_{1}, \ldots, \nu_{q}\right) \quad \text { of } \quad\{0, \ldots, p+q-1\}
$$

such that

$$
\mu_{1}<\ldots<\mu_{p} \text { and } \nu_{1}<\ldots<\nu_{q}
$$

and $(-1)^{(\mu, \nu)}$ is the sign of the permutation $(\mu, \nu)$.

We then extend this bracket to the whole of $\left(N^{-1} L\right)^{n}$ by setting

$$
\llbracket \partial^{I} v, \partial^{I} w \rrbracket:=\partial^{I} \llbracket v, w \rrbracket .
$$

That $\left(N^{-1} L\right)^{n}$ is a cosimplicial Lie algebra follows from the properties of the Eilenberg-Mac Lane shuffle product, as explained in [11] or [8].

Now set

$$
\mathcal{E}(L)^{n}(A)=\exp \left(\left(N^{-1} L\right)^{n} \otimes \mathfrak{m}_{A}\right),
$$

making $\mathcal{E}(L)$ into a cosimplicial complex of group-valued functors. To make it an SDC, we must define a $*$ product. We do this as the Alexander-Whitney cup product

$$
g * h=\left(\partial^{m+n} \ldots \partial^{m+2} \partial^{m+1} g\right) \cdot\left(\partial^{0}\right)^{m} h,
$$

for $g \in \mathcal{E}(L)^{m}, h \in \mathcal{E}(L)^{n}$.

Proposition 4.10. There are canonical isomorphisms $\mathrm{H}^{i}(L) \rightarrow \mathrm{H}^{i}\left(\mathrm{C}^{\bullet}(\mathcal{E}(L))\right)$, where $\mathrm{C}^{\bullet}(\mathcal{E}(L))$ is the tangent space of $\mathcal{E}(L)$, and an equivalence $\mathfrak{D e f}_{L} \rightarrow \mathfrak{D e} \mathfrak{f}_{\mathcal{E}(L)}$.

Proof. The tangent space $\mathrm{C}^{\bullet}(\mathcal{E}(L))$ is just $N^{-1} L$, so $L \cong N N^{-1} L$ by the standard comparison between cosimplicial and cochain complexes. This gives the required isomorphism on cohomology.

We define the equivalence by sending $\omega \in \mathrm{MC}_{L}(A)$ to $\exp (\omega) \in \mathcal{E}^{1}(L)$. We need to show that this satisfies the Maurer-Cartan functor. Now, the Campbell-BakerHausdorff formula implies that

$$
\exp (\omega) * \exp (\omega)=\exp \left(\partial^{2} \omega\right) \cdot \exp \left(\partial^{0} \omega\right)=\exp \left(\partial^{2} \omega+\partial^{0} \omega+\frac{1}{2} \llbracket \partial^{2} \omega, \partial^{0} \omega \rrbracket+\ldots\right),
$$

with all the remaining terms being higher commutators involving $\llbracket \partial^{2} \omega, \llbracket \partial^{2} \omega, \partial^{0} \omega \rrbracket \rrbracket$ and $\llbracket \partial^{0} \omega, \llbracket \partial^{2} \omega, \partial^{0} \omega \rrbracket \rrbracket$. But

$$
\left[\left[\partial^{2} \omega,\left[\left[\partial^{2} \omega, \partial^{0} \omega\right]\right]\right]\right]=\left[\left[\partial^{2} \omega,[\omega, \omega]\right]\right]=0,
$$

and similarly for $\left[\left[\partial^{0} \omega,\left[\left[\partial^{2} \omega, \partial^{0} \omega\right]\right]\right]\right]$, ensuring that all higher terms vanish. Therefore

$$
\exp (\omega) * \exp (\omega)=\exp \left(\partial^{2} \omega+\partial^{0} \omega+\frac{1}{2}[\omega, \omega]\right)=\partial^{1} \exp (\omega),
$$

since $d \omega+\frac{1}{2}[\omega, \omega]=0$, so $\mathrm{MC}_{L} \rightarrow \mathrm{MC}_{\mathcal{E}(L)}$. Finally, we have an isomorphism $\mathrm{G}_{L} \rightarrow$ 
$\mathcal{E}^{0}(L)$, compatible with the gauge actions, giving a morphism $\mathfrak{D e f}_{L} \rightarrow \mathfrak{D e f}_{\mathcal{E}(L)}$. That this is an equivalence follows from comparing tangent and obstruction spaces.

4.3. SDCs. Fix an SDC $E$. Although each $E^{n}$ will only be pro-representable in the sense of [9] if $\operatorname{dim} \mathrm{C}(E)^{n}<\infty$, in general we may make use of the weaker notion of pro-Artinian pro-representability as in [1]. Throughout this section, only the weaker notion of pro-representability will be used. We then have the following lemma:

Lemma 4.11. Given a smooth homogeneous functor $F: \mathcal{C}_{\Lambda} \rightarrow$ Set, let $\left\{t_{i}: i \in I\right\}$ be a basis for $t_{F}$. Then $F$ is isomorphic to $\operatorname{Hom}_{\operatorname{pro}\left(\mathcal{C}_{\Lambda}\right)}(\mathcal{R},-)$, where $\mathcal{R}$ is the proArtinian completion of $\Lambda\left[T_{i}: i \in I\right]$, localised at $\left(T_{i}: i \in I\right)$, for formal symbols $T_{i}$. Note that, if $t_{F}$ is finite dimensional, this is just the expected ring $\Lambda\left[\left[T_{1}, \ldots, T_{m}\right]\right]$.

Proof. This is very similar to the proof of [9, Theorem 2.11]. A full proof in the analogous case of nilpotent Lie algebras (rather than Artinian rings) is given in [7, Theorem 2.24].

Thus an SDC $E$ is equivalent to a system of smooth local pro-Artinian rings $\mathcal{Q}_{\bullet}$, with the dual structures to those described in Section 1.1. In particular, since we are only considering the case $\Lambda=k$, we have canonical $\partial_{\omega_{0}}^{0}, \partial_{\omega_{0}}^{n+1}$. Explicitly:

Lemma 4.12. $\mathcal{Q}$. is a simplicial complex of smooth local pro-Artinian $k$-algebras (with residue field $k$ ), together with a co-associative comultiplication

$$
\rho_{m, n}:=*_{m, n}^{\sharp}: \mathcal{Q}_{m+n} \rightarrow \mathcal{Q}_{m} \hat{\otimes} \mathcal{Q}_{n},
$$

such that $\mathcal{Q}_{0} \rightarrow k$ is the co-unit, satisfying:

(1) $\left(\partial_{i} \otimes \mathrm{id}\right) \circ \rho_{m+1, n}=\rho_{m, n} \circ \partial_{i}$ for $i \leq m$,

(2) $\left(\mathrm{id} \otimes \partial_{i}\right) \circ \rho_{m, n+1}=\rho_{m, n} \circ \partial_{m+i}$ for $i \geq 1$,

(3) $\left(\partial_{m+1} \otimes \mathrm{id}\right) \circ \rho_{m+1, n}=\left(\mathrm{id} \otimes \partial_{0}\right) \circ \rho_{m, n+1}$,

(4) $\left(\sigma_{i} \otimes \mathrm{id}\right) \circ \rho_{m-1, n}=\rho_{m, n} \circ \sigma_{i}$,

(5) $\left(\mathrm{id} \otimes \sigma_{i}\right) \circ \rho_{m, n-1}=\rho_{m, n} \circ \sigma_{m+i}$.

Of course, given a system $\mathcal{Q}$. satisfying these conditions, we can clearly recover an $\mathrm{SDC}$ as $\operatorname{Hom}\left(\mathcal{Q}_{\bullet},-\right)$, so that we may regard this as an equivalent definition of an SDC.

Remark 4.13. We say that a simplicial complex $A_{\bullet}$ of local $\Lambda$-algebras is Artinian if it satisfies DCC on simplicial ideals. This is equivalent to saying that the algebras $A_{n}$ are Artinian, and the normalised complex $N\left(A_{\bullet}\right)$ is bounded. It is easy to see that $\mathcal{Q}_{\bullet}=\lim Q_{\bullet}$, the limit being over quotient complexes $\mathcal{Q}_{\bullet} \rightarrow Q_{\bullet}$, where $Q_{\bullet}$ is Artinian. Moreover, $\mathcal{Q}_{\bullet}$ is really just a shorthand for this inverse system. Thus, for any functor $F$, by $F\left(\mathcal{Q}_{\bullet}\right)$ we mean $\lim _{\longleftarrow} F\left(Q_{\bullet}\right)$. In particular, $\mathrm{H}_{*}\left(\mathcal{Q}_{\bullet}\right):=\lim _{\leftarrow} \mathrm{H}_{*}\left(Q_{\bullet}\right)$. We will apply the same convention to the limits of DG complexes in the next section.

4.4. SDC $\rightsquigarrow$ DGLA. Given $\mathcal{Q}_{*}$ as in Section 4.3, we form a DG Hopf algebra $C(\mathcal{Q})$, with $C(\mathcal{Q})_{n}=\mathcal{Q}_{n}$ as a graded module. We let

$$
d=\sum_{i=0}^{n+1}(-1)^{i} \partial_{i}: C(\mathcal{Q})_{n+1} \rightarrow C(\mathcal{Q})_{n}
$$


and give it an algebra structure via the Eilenberg-Zilber shuffle product: for $x \in$ $C(\mathcal{Q})_{m}$ and $y \in C(\mathcal{Q})_{n}$, let

$$
x \nabla y=\sum_{(\mu, \nu) \in \operatorname{Sh}(m, n)}(-1)^{(\mu, \nu)}\left(\sigma_{\nu_{n}} \ldots \sigma_{\nu_{1}} x\right) \cdot\left(\sigma_{\mu_{m}} \ldots \sigma_{\mu_{1}} y\right) .
$$

As explained in [11] or [8], the resulting product is supercommutative, i.e.

$$
x \nabla y=(-1)^{m n} y \nabla x,
$$

and associative, and $d$ is a graded derivation with respect to $\nabla$, i.e.

$$
d(x \nabla y)=(d x) \nabla y+(-1)^{m} x \nabla(d y) .
$$

Lemma 4.14. $C(\mathcal{Q})_{*}$ is a Hopf algebra under the map $\rho$.

Proof. Given $x \in C(\mathcal{Q})_{m}, y \in C(\mathcal{Q})_{n}$, and any $p+q=m+n$, we have:

$$
\begin{aligned}
& \rho_{p, q}(x \nabla y)= \rho_{p, q}\left(\sum_{(\mu, \nu)}(-1)^{(\mu, \nu)} \sigma_{\nu_{n}} \ldots \sigma_{\nu_{1}} x \cdot \sigma_{\mu_{m}} \ldots \sigma_{\mu_{1}} y\right) \\
&= \sum_{(\mu, \nu)}(-1)^{(\mu, \nu)} \rho_{p, q}\left(\sigma_{\nu_{n}} \ldots \sigma_{\nu_{1}} x\right) \cdot \rho_{p, q}\left(\sigma_{\mu_{m}} \ldots \sigma_{\mu_{1}} y\right) \\
&= \sum \pm\left(\sigma_{\nu_{n^{\prime}}^{\prime}} \ldots \sigma_{\nu_{1}^{\prime}} \otimes \sigma_{\nu_{n^{\prime \prime}}^{\prime \prime}} \ldots \sigma_{\nu_{1}^{\prime \prime}}\right) \circ \rho_{m^{\prime}, m^{\prime \prime}}(x) \\
& \cdot\left(\sigma_{\mu_{m^{\prime}}^{\prime}} \ldots \sigma_{\mu_{1}^{\prime}} \otimes \sigma_{\mu_{m^{\prime \prime}}^{\prime \prime}} \ldots \sigma_{\mu_{1}^{\prime \prime}}\right) \circ \rho_{n^{\prime}, n^{\prime \prime}}(y),
\end{aligned}
$$

the final sum being over $m^{\prime}+m^{\prime \prime}=m, n^{\prime}+n^{\prime \prime}=n, m^{\prime}+n^{\prime}=p, m^{\prime \prime}+n^{\prime \prime}=q$, $\left(\mu^{\prime}, \nu^{\prime}\right) \in \operatorname{Sh}\left(m^{\prime}, n^{\prime}\right),\left(\mu^{\prime \prime}, \nu^{\prime \prime}\right) \in \operatorname{Sh}\left(m^{\prime \prime}, n^{\prime \prime}\right)$, with $\pm=(-1)^{\left(\mu^{\prime}, \mu^{\prime \prime}+p, \nu^{\prime}, \nu^{\prime \prime}+p\right)}$.

For this map to be a Hopf algebra map, we would need this expression to equal

$$
\sum \rho_{m^{\prime}, m^{\prime \prime}}(x) \nabla \rho_{n^{\prime}, n^{\prime \prime}}(y)
$$

the $(p, q)$ component of $\rho(x) \nabla \rho(y)$, summing over $m^{\prime}, m^{\prime \prime}, n^{\prime}, n^{\prime \prime}$ as above. Now,

$$
\begin{array}{r}
\rho_{m^{\prime}, m^{\prime \prime}}(x) \nabla \rho_{n^{\prime}, n^{\prime \prime}}(y)=\sum \pm\left(\sigma_{\nu_{n^{\prime}}^{\prime}} \ldots \sigma_{\nu_{1}^{\prime}} \otimes \sigma_{\nu_{n^{\prime \prime}}^{\prime \prime}} \ldots \sigma_{\nu_{1}^{\prime \prime}}\right) \circ \rho_{m^{\prime}, m^{\prime \prime}}(x) \\
\cdot\left(\sigma_{\mu_{m^{\prime}}^{\prime}} \ldots \sigma_{\mu_{1}^{\prime}} \otimes \sigma_{\mu_{m^{\prime \prime}}^{\prime \prime}} \ldots \sigma_{\mu_{1}^{\prime \prime}}\right) \circ \rho_{n^{\prime}, n^{\prime \prime}}(y)
\end{array}
$$

where the sum is over $\left(\mu^{\prime}, \nu^{\prime}\right),\left(\mu^{\prime \prime}, \nu^{\prime \prime}\right)$, and $\pm=(-1)^{m^{\prime \prime} n^{\prime}}(-1)^{\left(\mu^{\prime}, \nu^{\prime}\right)}(-1)^{\left(\mu^{\prime \prime}, \nu^{\prime \prime}\right)}$, the $(-1)^{m^{\prime \prime} n^{\prime}}$ arising from the (required) supercommutativity of $C(\mathcal{Q})_{*} \otimes C(\mathcal{Q})_{*}$. The result now follows from the observation that

$$
(-1)^{\left(\mu^{\prime}, \mu^{\prime \prime}+p, \nu^{\prime}, \nu^{\prime \prime}+p\right)}=(-1)^{m^{\prime \prime} n^{\prime}}(-1)^{\left(\mu^{\prime}, \nu^{\prime}\right)}(-1)^{\left(\mu^{\prime \prime}, \nu^{\prime \prime}\right)} .
$$

Lemma 4.15. $\rho$ is a chain map. 
Proof. For $x \in C(\mathcal{Q})_{m+1}$,

$$
\begin{aligned}
\rho(d x)= & \sum_{p+q=m} \rho_{p, q}\left(\sum_{i=0}^{m+1}(-1)^{i} \partial_{i} x\right) \\
= & \sum_{p+q=n}\left(\sum_{i=0}^{p}(-1)^{i}\left(\partial_{i} \otimes \mathrm{id}\right) \circ \rho_{p+1, q}(x)\right. \\
& \left.+(-1)^{p} \sum_{j=1}^{q+1}(-1)^{j}\left(\mathrm{id} \otimes \partial_{j}\right) \circ \rho_{p, q+1}(x)\right) \\
= & \sum_{p+q=m}\left(\left(d-(-1)^{p+1} \partial_{p+1}\right) \otimes \mathrm{id}\right) \circ \rho_{p+1, q}(x) \\
= & \sum_{p+q=m}(d \otimes \mathrm{id}) \circ \rho_{p+1, q}(x)-(-1)^{p+1}\left(\partial_{p+1} \otimes \mathrm{id}\right) \circ \rho_{p+1, q}(x) \\
& +\sum_{p+q=m}(-1)^{p}(\mathrm{id} \otimes d) \circ \rho_{p, q+1}(x)-(-1)^{p}\left(\mathrm{id} \otimes \partial_{0}\right) \circ \rho_{p, q+1}(x) \\
= & \sum_{p+q=m}(d \otimes \mathrm{id}) \circ \rho_{p+1, q}(x)+\sum_{p+q=m}(-1)^{p}(\mathrm{id} \otimes d) \circ \rho_{p, q+1}(x) \\
= & d \rho(x) .
\end{aligned}
$$

There is now a problem with $C\left(\mathcal{Q}_{*}\right)$ as a candidate to define a DGLA $L$ - it is not local. In [8], rather than taking the unnormalised chain complex $C\left(\mathcal{Q}_{*}\right)$, Quillen takes the normalised chain complex $N\left(\mathcal{Q}_{*}\right)$. Recall that

$$
\begin{gathered}
C(\mathcal{Q})_{n}=N(\mathcal{Q})_{n} \oplus D(\mathcal{Q})_{n}, \text { where } \\
N(\mathcal{Q})_{n}=\bigcap_{i=1}^{n} \operatorname{ker}\left(\partial_{i}: \mathcal{Q}_{n} \rightarrow \mathcal{Q}_{n-1}\right), \quad D(\mathcal{Q})_{n}=\sum_{i=1}^{n-1} \sigma_{i}\left(\mathcal{Q}_{n-1}\right) .
\end{gathered}
$$

Observe that $N(\mathcal{Q}) \bullet$ is local, as $\operatorname{ker}\left(\partial_{1}\right) \subset \mathfrak{m}_{\mathcal{Q}}$. It is pro-Artinian following Remark 4.13 , since the normalisation of an ideal is a $\nabla$-ideal, and $N(I) \nabla N(J) \subset N(I J)$. However, we cannot give $N(\mathcal{Q})$ the Hopf algebra structure $\rho$ : in general, for $x \in$ $N(\mathcal{Q})_{m+n},\left(\partial_{m} \otimes\right.$ id $) \circ \rho_{m, n}(x) \neq 0$. Instead, we take the complex

$$
\bar{N}(\mathcal{Q}):=C(\mathcal{Q}) / D(\mathcal{Q}) .
$$

It follows immediately from the identities in Lemma 4.12 that

$$
\rho_{m, n}: \bar{N}(\mathcal{Q})_{m+n} \rightarrow \bar{N}(\mathcal{Q})_{m} \hat{\otimes} \bar{N}(\mathcal{Q})_{n}
$$

is well defined. We need only check that $\nabla$ is well defined on $\bar{N}(\mathcal{Q})$, since then we will have $\bar{N}(\mathcal{Q})$ isomorphic to $N(\mathcal{Q})$ as an algebra.

Lemma 4.16. For $x \in D(\mathcal{Q})_{m}$ and $y \in C(\mathcal{Q})_{n}, x \nabla y \in D(\mathcal{Q})_{m+n}$.

Proof. Without loss of generality, $x=\sigma_{r} u$, for some $0 \leq r<m$. We will show that each term in the sum of the shuffle product is in $D(\mathcal{Q})_{m+n}$. Consider

$$
\sigma_{\nu_{n}} \ldots \sigma_{\nu_{1}} x \cdot \sigma_{\mu_{m}} \ldots \sigma_{\mu_{1}} y
$$


and let $a:=\max \left\{j: \nu_{j}-j<r\right\}$, which makes sense since $\nu_{j}-j$ is a non-decreasing function. With the obvious convention if this set is empty, we have $0 \leq a \leq n$. Now, the simplicial identities give

with

$$
\sigma_{\nu_{n}} \ldots \sigma_{\nu_{1}} \sigma_{r} u=\sigma_{\nu_{n}} \ldots \sigma_{\nu_{a+1}} \sigma_{r+a} \sigma_{\nu_{a}} \ldots \sigma_{\nu_{1}} u
$$

$$
\nu_{1}<\ldots<\nu_{a}<r+a<\nu_{a+1}<\ldots<\nu_{n} .
$$

Now $r+a \leq p-1+q$, and $r+a$ does not equal any of the $\nu_{j}$, so $r+a=\mu_{i}$ for some $i$. This gives

$$
\begin{aligned}
\left(\sigma_{\nu_{n}} \ldots \sigma_{\nu_{1}} x\right) \cdot\left(\sigma_{\mu_{m}} \ldots \sigma_{\mu_{1}} y\right)= & \left(\sigma_{\nu_{n}} \ldots \sigma_{\nu_{a+1}} \sigma_{\mu_{i}} \sigma_{\nu_{a}} \ldots \sigma_{\nu_{1}} u\right) \cdot\left(\sigma_{\mu_{m}} \ldots \sigma_{\mu_{1}} y\right) \\
= & \left(\sigma_{\mu_{i}} \sigma_{\nu_{n}-1} \ldots \sigma_{\nu_{a+1}-1} \sigma_{\nu_{a}} \ldots \sigma_{\nu_{1}} u\right) \\
& \cdot\left(\sigma_{\mu_{i}} \sigma_{\mu_{m}-1} \ldots \sigma_{\mu_{i+1}-1} \sigma_{\mu_{i-1}} \ldots \sigma_{\mu_{1}} y\right) \\
= & \sigma_{\mu_{i}}\left(\left(\sigma_{\nu_{n}-1} \ldots \sigma_{\nu_{a+1}-1} \sigma_{\nu_{a}} \ldots \sigma_{\nu_{1}} u\right)\right. \\
& \left.\cdot\left(\sigma_{\mu_{m}-1} \ldots \sigma_{\mu_{i+1}-1} \sigma_{\mu_{i-1}} \ldots \sigma_{\mu_{1}} y\right)\right)
\end{aligned}
$$

which is in $D(\mathcal{Q})_{m+n}$, as required.

Definition 4.17. Given an SDC $E$, we define the DGLA $\mathcal{L}(E)$ by

$$
\mathcal{L}(E)=\operatorname{Der}_{k}(\bar{N}(\mathcal{Q}) \bullet, k),
$$

where $\mathcal{Q}$. pro-represents $E$. Note that it follows from Lemma 4.9 that on $\mathcal{C}_{k}^{\mathbb{N}_{0}}$, $\bar{N}(\mathcal{Q})$ pro-represents $\exp (\mathcal{L}(E))$, so it must be a smooth, i.e. a graded power series ring.

Proposition 4.18. There are natural isomorphisms $\mathrm{H}^{i}(E) \rightarrow \mathrm{H}^{i}(\mathcal{L}(E))$.

Proof. We may compute $\mathrm{H}^{i}(E)$ using the normalised cocomplex

$$
N^{n}\left(K^{*}\right)=\bigcap_{i=0}^{n-1} \operatorname{ker}\left(\sigma^{i}: K^{n} \rightarrow K^{n-1}\right), \quad d=\sum_{i=0}^{n}(-1)^{i} \partial^{i} .
$$

Thus an element $\alpha \in N^{i}\left(\mathrm{C}^{\bullet}(E)\right)$ is a derivation (with respect to the product on $\mathcal{Q}$ ) from $\bar{N}_{i}(\mathcal{Q})$ to $k$ (by the definition of $\bar{N}$ ). A derivation to $k$ is just a linear map which annihilates both $k$ and $\mathfrak{m}_{\mathcal{Q}}^{2}$, but $\mathfrak{m}_{\mathcal{Q}} \nabla \mathfrak{m}_{\mathcal{Q}} \subset \mathfrak{m}_{\mathcal{Q}}^{2}$, so $\alpha$ gives a derivation (with respect to $\nabla$ ) from $\bar{N}_{\bullet}(\mathcal{Q})$ to $k$, of degree $i$. This defines a chain map, giving the required map on cohomology.

To see that these maps are isomorphisms, consider the morphism of filtered complexes

$$
\left(N_{\bullet}(\mathcal{Q}), N_{\bullet}\left(\mathfrak{m}_{\mathcal{Q}}\right)^{\nabla n}\right) \stackrel{f}{\rightarrow}\left(\mathcal{Q}_{\bullet}, \mathfrak{m}_{\mathcal{Q}}^{n}\right) .
$$

The spectral sequences associated to the filtrations are

$$
\begin{aligned}
& E_{p q}^{1}=\left(\operatorname{Symm}^{-p} \mathrm{H}_{*}(\mathcal{L}(E))\right)_{p+q} \quad \Longrightarrow \quad \mathrm{H}_{p+q}\left(N_{\bullet}(\mathcal{Q})\right), \\
& E_{p q}^{1}=\left(\operatorname{Symm}^{-p} \mathrm{H}_{*}(E)\right)_{p+q} \quad \Longrightarrow \quad \mathrm{H}_{p+q}\left(\mathcal{Q}_{\bullet}\right),
\end{aligned}
$$

where we write $\mathrm{H}_{*}(E)$ for the pro-Artinian dual of $\mathrm{H}^{*}(E)$, and likewise for $\mathrm{H}_{*}(\mathcal{L}(E))$. The expression for the $E^{1}$ term follows from the calculation of homology of symmetric powers of chain complexes and simplicial complexes.

If $i$ is the smallest integer for which $\mathrm{H}^{i}(f): \mathrm{H}^{i}(E) \rightarrow \mathrm{H}^{i}(\mathcal{L}(E))$ is not an isomorphism, then $E_{i}^{\infty}(f)$ is not an isomorphism. This gives a contradiction, since the morphism of spectral sequences is an isomorphism at the $E^{\infty}$ term, so the maps $\mathrm{H}^{i}(f)$ are all isomorphisms. 
Proposition 4.19. There is a natural map $\mathfrak{D e f}_{E} \rightarrow \mathfrak{D e f}_{\mathcal{L}(E)}$, with the maps on cohomology compatible with the corresponding maps on tangent and obstruction spaces.

Proof. (1) Given $\omega \in \mathrm{MC}_{E}$, we may regard $\omega$ as a continuous ring homomorphism $\omega: \mathcal{Q}_{1} \rightarrow A$, satisfying $\omega * \omega=\partial^{1}(\omega)$. We also know that $\sigma^{0}(\omega)=e$. Set $\alpha=\omega-\omega_{0}$. This gives $\sigma^{0}(\alpha)=0$; therefore $\alpha: \bar{N}_{1}(\mathcal{Q}) \rightarrow A$. In fact, $\alpha$ is a $\nabla$-derivation: for $x \in \mathcal{Q}_{0}, y \in \mathcal{Q}_{1}$,

$$
\begin{aligned}
\alpha(x \nabla y) & =\alpha\left(\sigma_{0}(x) y\right) \\
& =\left(\omega-\omega_{0}\right)\left(\sigma_{0}(x) y\right) \\
& =\sigma^{0}(\omega)(x) \bar{y}-\sigma^{0}\left(\omega_{0}\right)(x) \bar{y}+\overline{\sigma_{0}(x)} \omega(y)-\overline{\sigma_{0}(x)} \omega_{0}(y) \\
& =\bar{x} \alpha(y) \\
& =\bar{x} \alpha(y)+\alpha(x) \bar{y} .
\end{aligned}
$$

Moreover, the Maurer-Cartan equation for $\omega$ becomes

$$
\begin{aligned}
\left(\omega_{0}+\alpha\right) *\left(\omega_{0}+\alpha\right) & =\partial^{1}\left(\omega_{0}+\alpha\right), \\
\omega_{0}^{2}+\partial^{0}(\alpha)+\partial^{2}(\alpha)+\alpha * \alpha & =\omega_{0}^{2}+\partial^{1}(\alpha), \\
\partial^{0}(\alpha)-\partial^{1}(\alpha)+\partial^{2}(\alpha)+\alpha * \alpha & =0 \\
d \alpha+\frac{1}{2}[\alpha, \alpha] & =0 .
\end{aligned}
$$

Therefore $\alpha \in \mathrm{MC}_{\mathcal{L}(E)}$.

(2) Given $g \in E^{0}(A), g$ is an algebra homomorphism $g: \mathcal{Q}_{0} \rightarrow A$. Since $\nabla$ agrees with the usual product on $\mathcal{Q}_{0}$, and $\bar{N}_{0}(\mathcal{Q})=\mathcal{Q}_{0}, g$ is a $\nabla$-algebra homomorphism $g: \bar{N}_{0}(\mathcal{Q}) \rightarrow A$. Therefore

$$
g \in \exp \left(\mathcal{L}^{0}(E) \otimes \mathfrak{m}_{A}\right)
$$

by Lemma 4.9. It is easy to see that the adjoint action on $E$ corresponds to the gauge action on $\mathcal{L}(E)$.

We therefore have the map $\mathfrak{D e f}_{E} \rightarrow \mathfrak{D e f}_{\mathcal{L}(E)}$. The results on tangent and obstruction spaces follow immediately.

\subsection{The equivalence.}

Theorem 4.20. There is a quasi-isomorphism $E \rightarrow \mathcal{E}(\mathcal{L}(E))$ of $S D C$ s, for any $S D C E$.

Proof. By Lemma 4.9, we see that the Hopf algebra $N_{\bullet}(\mathcal{Q})$ must be the free proArtinian algebra generated by the pro-Artinian dual $\mathcal{L}(E)^{\vee}$ of $\mathcal{L}(E)$. Letting $N^{-1}$ denote the denormalisation functor from chain complexes to simplicial complexes, we then have a canonical map $N^{-1} \mathcal{L}(E)^{\vee} \rightarrow \mathcal{Q}$. of simplicial complexes, and therefore a map $k\left[\left[N^{-1} \mathcal{L}(E)^{\vee}\right]\right] \rightarrow \mathcal{Q}$. The former algebra pro-represents $\mathcal{E}(\mathcal{L}(E))$, so we have defined a morphism $E \rightarrow \mathcal{E}(\mathcal{L}(E))$, respecting the operations $\sigma^{i}, \partial^{i}$. It follows from Propositions 4.10 and 4.18 that this map is an isomorphism on cohomology. 
It only remains to verify that this map respects the product $*$, which follows because the coproduct on Hopf algebras is not changed by any of these constructions, the Alexander-Whitney and Eilenberg-Zilber maps cancelling each other.

Alternatively, we could observe that, if $\mathcal{T}_{\bullet}$ is the DG algebra pro-representing $\exp (L)$, then $\mathcal{E}(L)$ is pro-represented by $N^{*} \mathcal{T}_{\bullet}$, for $N^{*}$ the denormalisation functor defined for algebras in [8]. The morphism required is just the co-unit of the adjunction $N^{*} \dashv N$, which is natural with respect to the coproduct.

Corollary 4.21. For any $D G L A L$, there is a quasi-isomorphism $L \rightarrow \mathcal{L}(\mathcal{E}(L))$.

Proof. By Theorem 4.20, there is a quasi-isomorphism of SDCs

$$
\mathcal{E}(L) \rightarrow \mathcal{E}(\mathcal{L}(\mathcal{E}(L))) .
$$

Now, Theorem 4.10 gives a canonical isomorphism $N(\mathrm{C}(\mathcal{E}(L))) \cong L$, so we may regard $N^{\bullet}(\mathrm{C}(\mathcal{E}(L)))$ as a DGLA, with bracket

$$
[\alpha, \beta]=(\alpha \otimes \beta) \circ \Delta \mp(\beta \otimes \alpha) \circ \Delta .
$$

(Note that $N(\mathrm{C}(E))$ will not be closed under this bracket for an arbitrary SDC $E$.)

The quasi-isomorphism of SDCs then gives us a quasi-isomorphism of DGLAs:

$$
L \cong N(\mathrm{C}(\mathcal{E}(L))) \stackrel{\sim}{\longrightarrow} N(\mathrm{C}(\mathcal{E}(\mathcal{L}(\mathcal{E}(L))))) \cong \mathcal{L}(\mathcal{E}(L)) .
$$

If we now define SDC to be the category of SDCs, localised at quasi-isomorphisms, DGLA to be the category of DGLAs, localised at quasi-isomorphisms, and $\left[\mathcal{C}_{\Lambda}, \mathrm{Grpd}\right]$ the category of groupoid-valued functors on $\mathcal{C}_{\Lambda}$, localised at equivalences, we have defined a pair of equivalences of fibred categories

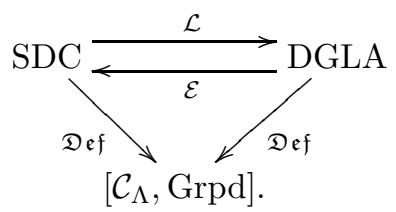

\section{REFERENCES}

[1] Alexander Grothendieck. Technique de descente et théorèmes d'existence en géométrie algébrique. II. Le théorème d'existence en théorie formelle des modules. In Séminaire Bourbaki, Vol. 5, Exp. No. 195, pages 369-390. Soc. Math. France, Paris, 1995. MR1603480

[2] Vladimir Hinich. Deformations of sheaves of algebras. Adv. Math., 195(1):102-164, 2005. MR2145794

[3] Luc Illusie. Complexe cotangent et déformations. I. Lecture Notes in Mathematics, Vol. 239, Springer-Verlag, Berlin, 1971. MR0491680 (58:10886a)

[4] Saunders Mac Lane. Categories for the working mathematician. Graduate Texts in Mathematics, Vol. 5, Springer-Verlag, New York, 1971. MR0354798 (50:7275)

[5] Marco Manetti. Deformation theory via differential graded Lie algebras. In Algebraic Geometry Seminars, 1998-1999 (Italian) (Pisa), pages 21-48. Scuola Norm. Sup., Pisa, 1999. arXiv math.AG/0507284. MR1754793

[6] J. P. Pridham. Deforming $l$-adic representations of the fundamental group of a smooth variety. J. Algebraic Geom., 15(3):415-442, 2006. MR2219844

[7] J. P. Pridham. The pro-unipotent radical of the pro-algebraic fundamental group of a compact Kähler manifold. Ann. Fac. Sci. Toulouse Math., 6, 16(1):147-178.

[8] Daniel Quillen. Rational homotopy theory. Ann. of Math. (2), 90:205-295, 1969. MR0258031 $(41: 2678)$ 
[9] Michael Schlessinger. Functors of Artin rings. Trans. Amer. Math. Soc., 130:208-222, 1968. MR0217093 (36:184)

[10] Moss E. Sweedler. Hopf algebras. Mathematics Lecture Note Series. W. A. Benjamin, Inc., New York, 1969. MR0252485 (40:5705)

[11] Charles A. Weibel. An introduction to homological algebra. Cambridge University Press, Cambridge, 1994. MR1269324 (95f:18001)

Trinity College, Cambridge, CB2 1TQ, United Kingdom

E-mail address: J.P.Pridham@dpmms.cam.ac.uk 